\title{
Stokes-Brinkman formulation for prediction of void formation in dual-scale fibrous reinforcements: a BEM/DR-BEM simulation
}

\author{
Iván David Patiño ${ }^{1}$ • Henry Power ${ }^{2}$ • César Nieto-Londoño ${ }^{3}$. Whady Felipe Flórez ${ }^{4}$
}

Received: 29 August 2016 / Accepted: 22 November 2016 / Published online: 16 February 2017

(C) The Author(s) 2017. This article is published with open access at Springerlink.com

\begin{abstract}
A numerical study of voids formation in dualscale fibrous reinforcements is presented. Flow fields in channels (Stokes) and tows (Brinkman) are solved via direct Boundary Element Method and Dual Reciprocity Boundary Element Method, respectively. The present approach uses only boundary discretization and Dual Reciprocity domain interpolation, which is advantageous in this type of moving boundary problems and leads to an accurate representation of the moving interfaces. A problem admitting analytical solution, previously solved by domain-meshing techniques, is used to assess the accuracy of the present approach, obtaining satisfactory results. Fillings of Representative Unitary Cells at constant pressure are considered to analyze the influence of
\end{abstract}

Henry Power

henry.power@nottingham.ac.uk

Iván David Patiño

ivandavidpatino@gmail.com

César Nieto-Londoño

cesar.nieto@upb.edu.co

Whady Felipe Flórez

whady.florez@upb.edu.co

1 Grupo de Investigación en Nuevos Materiales (GINUMA), Universidad Pontificia Bolivariana, Circular 1 Avenida 73-76, Bloque 22B, Piso 1, Medellín, Colombia

2 School of Mechanical, Materials and Manufacturing Engineering, University of Nottingham, Coates Building, University Park, Nottingham NG7 2DR, UK

3 Grupo de Ingeniería Aeroespacial, Universidad Pontificia Bolivariana, Circular 1ra No 73-34 (Bloque 22), Medellín, Colombia

4 Grupo de Energía y Termodinámica, Universidad Pontificia Bolivariana, Circular 1ra No 73-34 (Bloque 22), Medellín, Colombia capillary ratio, jump stress coefficient and two formulations (Stokes-Brinkman and Stokes-Darcy) on the filling process, void formation and void characterization. Filling times, fluid front shapes, void size and shape, time and space evolution of the saturation, are influenced by these parameters, but voids location is not.

Keywords Boundary Element Method - Stokes-Brinkman formulation - Stress matching conditions - Void formation . Dual-scale fibrous reinforcement

\section{Introduction}

In dual-scale fibrous reinforcements used in composites processing, the permeability of the tows is several orders of magnitudes lower than the permeability of the inter-tow channels. This causes imbalances between the flow inside the tows and the flow in the channels, which can lead to the formation of voids by mechanical entrapment of air. Once these voids are formed, several phenomena can take place, among others: bubble compression, displacement, migration and splitting.

Under the assumptions that the solid phase is stationary, the fluid is Newtonian and incompressible and neglecting the inertial effects, the Navier-Stokes system of equations governing the flow inside the pores of the porous medium (microscale) reduces to the Stokes equation. By an assembled average including both phases of the porous medium (liquid and solid), the Stokes system of equations reduces to Darcy's law, where the velocity (Darcy velocity) is linearly proportional to the pressure gradient, with proportionality constant given by the permeability, Darcy's law was established empirically by Darcy (1856) and later derived formally by different authors using several approaches (among others see Whitaker [66]). 
Considering the shearing effect between the fluid and the walls of the porous medium, Brinkman (1947) added the second-order derivatives of the velocity to the Darcy equation, resulting in the Brinkman equation. The averages implicit in the Brinkman equation can be viewed as averages over an ensemble of different scales of the porous medium that interpolate between the Stokes and Darcy equations. On small length scales, the pressure gradient balances the Laplacian of the velocity and the flow is essentially viscous, Stokes flow, while over larger length scales, the velocity is slowly varying and the pressure gradient balances the average velocity as it does in Darcy's law (for more details see Durlofsky and Brady [9]). According to Ochoa-Tapia and Whitaker [40], Brinkman approximation can be applied when the following three length scale constrains are simultaneously satisfied: $r_{0}^{2} /\left(l_{\varepsilon} l_{p}\right) \ll 1, r_{0}^{2} /\left(l_{\varepsilon} l_{v}\right) \ll 1, l_{f} \ll r_{0}$, where $l_{\varepsilon}, \quad l_{p}, l_{v}$ and $l_{f}$ are the characteristic lengths associated to the porosity, pressure, velocity gradients and fluid phase, respectively, and $r_{0}$ is the characteristic length scale of the Representative Elementary Volume (REV). Krotkiewski et al.[28] reported a direct numerical simulation of the flow field in homogeneous two dimensional porous media with characteristic length $L$ and permeability $K$, concluding that the Stokes solution is dominant for $K / L^{2} \geq 10$, Darcy law is representative of the flow field if $K / L^{2} \leq 10^{-4}$, while for $10^{-4} \leq K / L^{2} \leq 10$ the Brinkman approximation should be used to account for the transition between both flow regimes, Stokes and Darcy.

The Brinkman equation includes an effective viscosity term, $\mu_{e f f}$, to consider the viscous diffusion not deemed in the Darcy law. In order to explain the meaning of this term, it is necessary to consider that the volume averaging method allows describing the porous medium flow in terms of averages of the local quantities by means of the Darcy-Brinkman equation, which is given by (3) considering $\mu_{\text {eff }}=\mu$, where $\mu$ is the real fluid viscosity. This last equality is valid provided that a non-slip condition on the interfaces between the fluid and solid phases of the porous medium is considered, as done in the traditional volume-averaging method. However, the non-coincidence between $\mu_{\text {eff }}$ and $\mu$ has been demonstrated in several experimental, numerical and theoretical works, suggesting that the non-slip condition is not necessarily valid in all cases. For instance, Givler and Altobelli [17] experimentally found that $\mu_{\text {eff }}$ is 7.5 times the value of $\mu$ for high-porous open cell foams and moderate Reynolds numbers, whereas Starov and Zhdanov [70] studied the dependency of $\mu_{e f f}$ on the porosity and particle size in porous media composed of equally sized spherical particles, finding that $\mu_{\text {eff }}$ can be lower or larger than $\mu$. On the other hand, the numerical flow simulations conducted by [4] in 3D regular arrays of cubes showed that $\mu_{\text {eff }}<\mu$, whereas those ones executed by [34] indicated that $\mu_{\text {eff }}>\mu$ in order to match the Brinkman equation with the numerical solutions in the boundary layer developed in the porous medium domain when it is in contact with a free-fluid domain. A very illustrative theoretical work was published by [63], where it was demonstrated that the effective viscosity, $\mu_{e f f}$, is different to the fluid viscosity, $\mu$, when a slip condition at the fluidsolid interface of the porous medium is considered. Using an up-scaling procedure, a boundary-value problem to compute $\mu_{\text {eff }}$ was obtained in such work, achieving important conclusions. In general, the effective viscosity is different to the fluid viscosity, i.e, $\mu_{e f f} \neq \mu$, because it is a term that 'absorbs' the microscale variations of the velocity gradient when a variationless velocity gradient model is used at macroscopic scale. On the other hand, as the prescribed slip coefficient at the fluid-solid interface increases, the boundary layer thickness decreases and a non-slip condition tends to be reached, in such a way that in the limit when the slip coefficient tends to infinity, $\mu_{e f f}=\mu$.

In the Stokes-Brinkman approach the matching conditions at the interface channels-tows are defined in terms of the corresponding surface velocity and traction vectors. Continuity of the velocity field is always considered and two types of conditions could be employed for the tractions: continuous [39] and jump stress [40,41]. The continuity of stress was initially implemented in $[39,48]$, however a jump stress matching condition was proposed later in [40] based on the non-local form of the volume-averaged momentum equation for the analysis of the region between the channel and the porous medium. By means of experiments of unidirectional flow in parallel domains, Ochoa-Tapia and Whitaker [41] found that the jump stress tensor across the interface for isotropic porous media, $\left[\left|\sigma_{i j} \hat{n}_{j}\right|\right]$, is linearly proportional to the interface surface velocity $u_{j}$, i.e. $\left[\left|\sigma_{i j} n_{j}\right|\right]=$ $\mu T_{i j} u_{j} / \sqrt{K}$, where $\mu$ stands for the fluid viscosity, while the second-order tensor $T_{i j}$ is given by $\beta \delta_{i j}$, with $\beta$ as the jump stress coefficient ranging between -1 and 1.47; for more details see [23]. However, Angot [2], demonstrated mathematically that the well-posedness of the Stokes-Brinkman problem is only possible when $\beta \geq 0$. The theoretical estimation of the value of $\beta$ was developed in [64,65], where it was established that $\beta$ depends on the porosity of the porous medium in the inter-region channel-tow, $\varepsilon_{c t}$.

The estimation of the effective viscosity term, $\mu_{e f f}$, in the Brinkman equation is known to depend on the geometry of the porous medium and the flow itself. As suggested in the literature $[23,56,57]$, an acceptable approximation for $\mu_{\text {eff }}$ in dual-scale fibrous reinforcements is to consider that $\mu_{\text {eff }}=\mu$ when the continuous stress condition is used, whereas $\mu_{e f f}=\mu / \varepsilon_{t}$ is suitable when the jump stress condition is employed, with $\varepsilon_{t}$ as the tow porosity. In a recent work [23], focusing in the prediction of the effective saturated permeability of dual-scale reinforcements for unidirectional flow (where a value of $\beta=0.7$ was used), it was found that the type of stress matching condition (continuity or jump) 
has a strong influence on the boundary layer thickness in the porous medium, but not on the effective saturated permeability, which is dependent on the pore geometry. In this point, it is relevant to mention that the study of the pore geometrical dependency of the effective permeability could be a complicated and computational expensive task, but some recent efforts have been addressed to reduce the computational cost without compromising the accuracy. For instance, a multiscale framework relating the effective permeability with some microstructural attributes extracted by X-ray tomography was proposed in [55], where several computational techniques were efficiently combined (Level Set, Graph Theory and Lattice Boltzman/Finite Element) to determine the tortuosity, porosity and homogenized effective permeability at specimen scale. The particular problem solved in [23] by FEM is also considered here for validation of the numerical technique implemented.

In the present work, simulations of fillings of RUC's (Representative Unitary Cells) of dual-scale fibrous reinforcements are carried out using a Boundary Element/Dual Reciprocity numerical approach (BEM/DR-BEM). The flow in the channel, Stokes flow, is given by its boundary-only integral formulation, Green's formula, and numerically treated by direct BEM, while the flow inside the tows, Brinkman flow, is given by a boundary-domain integral formulation in terms of the Stokes fundamental solutions, where the domain integral is transformed into a boundary integral using the Dual Reciprocity Boundary Element Method (DR-BEM). The present numerical approach is validated by comparison with a benchmark analytical solution used previously in the literature to assess the robustness of FEM-based numerical solutions of flow problems in dual-scale porous media $[23,58]$.

The developed BEM/DR-BEM numerical scheme is then used to simulate the simultaneous filling of channels and tows inside the RUC at constant pressure regime with the purpose of analyzing the influence of the stress matching conditions on the void formation at several values of the capillary ratio, $C_{c a p}=P_{c a p, \max } / P_{i n}$, where $P_{c a p, \max }$ and $P_{i n}$ stand for the maximum capillary pressure and the inlet pressure, respectively. This type of filling problem has been previously considered in the literature using different formulations and numerical techniques. For instance, Gourichon et al. [19] studied the influence of the RUC porosity in the formation of voids using a Darcy-Darcy formulation and the FEM/CV conforming method. On the other hand, Schell et al. [49] studied the influence of the tow porosity, $\varepsilon_{t}$, on the final void content using the same formulation and numerical technique as in [19], while the problem of unidirectional filling of circular tows in cylindrical coordinates was analyzed in [68] using a Stokes-Brinkman formulation and the Finite Volume method, where it was studied the effect of the filling velocity, resin viscosity, inter-tow dimension and intra-tow dimension on the shape of the fluid front.

In comparison with domain-meshing numerical techniques previously used in the literature for the simulation of the filling process in dual scale fibrous reinforcements based on the Stokes-Brinkman formulation [11,23,50,51,58], two main advantages can be identified by the use of the BEM/DRBEM approach employed in this work. Firstly, the use of BEM-based techniques does not imply any mesh discretization of the problem domain, which is not a trivial task in a domain that is continuously changing in size and shape as in the present case. Secondly, the tracking of the fluid front is directly carried out by an Euler integration of the kinematic condition at the moving interface, which assures a higher order accuracy on the prediction of the fluid front shape without the need of implementing any additional reconstruction algorithm of the moving boundaries, contrary to the case of the Volume of Fluid (VOF) $[14,15]$ or the Level Set Method $[12,13,53]$.

Another important contribution of this work is the study of the influence of two types of stress matching conditions (continuous and jump) on the void formation. Other works have analyzed the influence of these conditions on the effective saturated permeability $[23,58]$, but not on the size, shape and location of the voids formed by mechanical entrapment of air. Additionally, the processes of the void formation using the Stokes-Brinkman and Stokes-Darcy formulations are compared each other, which has not been reported before to the best of our knowledge. Finally, the consideration of a flow direction-dependent capillary pressure in the tows without experimental factors and of the curvature- dependent surface traction effects for the fluid fronts in the channels are ones of the main differences between the present work and previous publications in the same field $[1,7,49,59]$.

\section{Governing equations, boundary and matching conditions}

In the Stokes-Brinkman formulation, the governing equations at each medium are defined as:

Mass conservation (For all domains):

$$
\frac{\partial u_{i}}{\partial x_{i}}=0
$$

Momentum for the Stokes domain (Channels flow):

$\mu\left(\frac{\partial^{2} u_{i}}{\partial x_{j} x_{j}}\right)-\frac{\partial p}{\partial x_{i}}=0$

Momentum for the Brinkman domain (Porous media or tows flow): 
$\mu_{e f f} \frac{\partial^{2} u_{i}}{\partial x_{j} \partial x_{j}}-\frac{\partial p}{\partial x_{i}}=\frac{\mu}{K_{i}} u_{i}$

where in the right hand side of (3) the double index notation is not considered for $i=1,2$. Here $u_{i}, p, \mu, \mu_{\text {eff }}$ and $K_{i}$ represent the velocity vector, pressure, liquid viscosity, effective viscosity and main permeabilities, respectively.

Let us define the following non-dimensional variables for the length, velocity, time and pressure [46,47]:

For all domains:

$$
\begin{aligned}
\widehat{x_{i}} & =x_{i} / L^{*} \\
\hat{u}_{i} & =u_{i} / U_{\max } \\
\hat{t} & =t /\left(L^{*} / U_{\max }\right)
\end{aligned}
$$

For the Stokes domain (Channels flow):

$\hat{p}=\frac{p}{\mu \cdot U_{\max } / L^{*}}$

For the Brinkman domain (Porous media or tows flow):

$\hat{p}=\frac{p}{\mu_{\text {eff }} \cdot U_{\max } / L^{*}}$

where $L^{*}$ and $U_{\max }$ are the characteristic length and the maximum velocity of the problem, respectively. In terms of these characteristic values, the non-dimensional form of the governing Eqs. (1), (2), (3) can be written as:

$$
\begin{aligned}
& \frac{\partial \hat{u}_{i}}{\partial \hat{x}_{i}}=0 \\
& \frac{\partial^{2} \hat{u}_{i}}{\partial \hat{x}_{j} \hat{x}_{j}}-\frac{\partial \hat{p}}{\partial \hat{x}_{i}}=0 \\
& \frac{\partial^{2} \hat{u}_{i}}{\partial \hat{x}_{j} \partial \hat{x}_{j}}-\frac{\partial \hat{p}}{\partial \hat{x}_{i}}=\chi_{i}^{2} \hat{u}_{i}
\end{aligned}
$$

where $\chi_{i}^{2}=1 / D a_{i}^{*}=\left(L^{*}\right)^{2} \mu /\left(K_{i} \cdot \mu_{\text {eff }}\right)$ is the inverse of the Darcian number, $D a_{i}^{*}$, in the principal direction $i$.

The non-dimensional matching conditions for the StokesBrinkman problem are as follows [2]:

- Continuity of velocities:

$$
\hat{u}_{i}^{(s)}=\hat{u}_{i}^{(b)}
$$

- Normal and tangential component of the jump stress:

$$
\begin{aligned}
& \left(\hat{t}_{i}^{(s)}+\left(\mu_{e f f} / \mu\right) \hat{t}_{i}^{(b)}\right) \hat{n}_{i}=-\frac{L^{*}}{\sqrt{K_{n}}} \beta_{n} \hat{u}_{i}^{(s)} \hat{n}_{i} \\
& \left(\hat{t}_{i}^{(s)}+\left(\mu_{e f f} / \mu\right) \hat{t}_{i}^{(b)}\right) \hat{\tau}_{i}=-\frac{L^{*}}{\sqrt{K_{\tau}}} \beta_{\tau} \hat{u}_{i}^{(s)} \hat{\tau}_{i}
\end{aligned}
$$

where $s$ and $b$ represent the Stokes and Brinkman domain, respectively, and $\hat{t}_{i}^{(s)}=\left(L^{*} /\left(\mu U_{\max }\right)\right) \sigma_{i j} \widehat{n_{j}}$ and $\hat{t}_{i}^{(b)}=$ $\left(L^{*} /\left(\mu_{e f f} U_{\max }\right)\right) \sigma_{i j} \widehat{n_{j}}$ are defined as the dimensionless traction vectors, with $\sigma_{i j}$ as the Cauchy stress tensor. Here $K_{n}$ and $K_{\tau}$ are the effective permeabilities in the normal and tangential direction, respectively, with the effective permeability in any orientation, $\phi_{i}$, given by [33]:

$K_{e f f}\left(\phi_{i}\right)=\frac{K_{1} \cdot K_{2}}{K_{1} \cdot \sin ^{2}\left(\phi_{i}\right)+K_{2} \cdot \cos ^{2}\left(\phi_{i}\right)}$

where $\phi_{i}$ is the angle between a given direction and the major axis of permeability. In the above jump stress conditions, $\beta_{n}$ and $\beta_{\tau}$ are the normal and tangential jump coefficients, which are considered equal in the present work as done in $[23,58]$. Some authors consider that the minus sign of the right hand side of (13) and (14) is implicit in the stress jump coefficients, $\beta_{n}$ and $\beta_{\tau}[23,58]$. When a continuous surface traction at the interface is considered, the corresponding matching condition can be obtained from (13) and (14) by setting the values of $\beta_{n}$ and $\beta_{\tau}$ to zero.

In the present paper, the RUC filling is carried out at constant inlet pressure for all cases. Therefore, the nondimensional inlet boundary conditions are as follows:

At the Stokes domain:

$\hat{t}_{1}^{(s)}=-\bar{p} L^{*} /\left(\mu U_{\max }\right) \widehat{n_{1}^{(s)}}, \hat{u}_{2}^{(s)}=0$

At the Brinkman domain:

$\hat{t}_{1}^{(b)}=-\bar{p} L^{*} /\left(\mu_{e f f} U_{\max }\right) \widehat{n_{1}^{(b)}}, \hat{u}_{2}^{(b)}=0$

where $\bar{p}$ represents the prescribed inlet pressure. No-flux condition, $\hat{u}_{i} \hat{n}_{i}=0$, and zero traction in the tangential direction, $\hat{t}_{i} \hat{\tau}_{i}=0$, are used in boundaries where symmetry is specified.

At the fluid fronts, kinematic and dynamic boundary conditions are defined. The former condition establishes that the fluid front advances along its normal direction, while the dynamic condition accounts for the discontinuities of normal stress due to the capillary pressure, $p_{c a p}$. Both kind of conditions are represented in (18) and (19), (20), respectively:

- Kinematic condition (For all domains):

$$
d \hat{x}_{i} / d \hat{t}=\hat{u}_{n} \widehat{n_{i}}=\left(\hat{u}_{j} \cdot \widehat{n_{j}}\right) \widehat{n_{i}}
$$

- Dynamic condition (At the Stokes domain):

$$
\hat{t}_{i}^{(s)}=L^{*}\left(p_{c a p}-p_{a}\right) /\left(\mu U_{\text {max }}\right) \widehat{n_{i}}
$$

- Dynamic condition (At the Brinkman domain):

$$
\hat{t}_{i}^{(b)}=L^{*}\left(p_{\text {cap }}-p_{a}\right) /\left(\mu_{e f f} U_{\text {max }}\right) \widehat{n_{i}}
$$


where $\widehat{n_{i}}, \quad p_{a}$ and $\hat{u}_{n}$ are the outwardly oriented normal vector, air pressure and dimensionless normal velocity at the fluid front, respectively. In (18), $\hat{u}_{n}$ is given by the Stokes velocity in the channel domain and by the pore velocity in the porous medium, which, in turn, is defined as the Brinkman velocity divided by the tow porosity, $\varepsilon_{t}[32,67]$. In the case of the channel flow, the capillary pressure is $p_{\text {cap }}=-\sigma \kappa=$ $-\sigma\left(\partial \hat{n}_{i} / \partial x_{i}\right)$, with $\sigma$ as the surface tension and $\kappa$ as the curvature of the moving boundary. For the flow inside the porous medium, the capillary pressure can be computed using the following equation [35]:

$$
\begin{aligned}
p_{\text {cap }} & =2\left(\sigma \cdot \cos (\theta) / R_{e c}\right) \\
R_{e c} & =2\left(A_{\text {int }, s} / C_{\text {int }, s}\right)\left(\varepsilon_{t} /\left(1-\varepsilon_{t}\right)\right)
\end{aligned}
$$

where $A_{i n t, s}$ is the cross sectional area of the solid particles in the flow direction, $C_{i n t, s}$ is the wetted perimeter of the solid particles, $\theta$ is the contact angle and $\varepsilon_{t}$ is the tow porosity. In this work, the geometry of the porous medium is considered as a bank of aligned circular fibers and the capillary pressure in any point of the longitudinal tow (warp) depends on the angle between the normal of the fluid front and the principal axis of the fibers, $\varphi$. If the flow infiltrates the warp in parallel, $\varphi=0$, or oblique direction, $0<\varphi<\pi / 2, A_{i n t, s}$ and $C_{i n t, s}$ are approximated as the cross sectional area and perimeter of a truncated circular cylinder using Gauss-Kummer series for the perimeter of the resulting ellipse [5], as given by:

$$
\begin{aligned}
A_{i n t, s}= & n_{f} \pi R^{2} \sec (\varphi) \\
C_{i n t, s}= & n_{f} \pi R(1+\sec (\varphi)) \\
& \times\left(1+\sum_{i=1}^{\infty}\left(\begin{array}{c}
1 / 2 \\
i
\end{array}\right)\left(\frac{\sec (\varphi)-1}{\sec (\varphi)+1}\right)^{2 i}\right)
\end{aligned}
$$

If the impregnation occurs perpendicular to the fibers, $\varphi=$ $\pi / 2$, the following equations are used:

$$
\begin{aligned}
& A_{i n t, s}=2 n_{f} L_{f} R \\
& C_{i n t, s} \approx 2 n_{f} L_{f}
\end{aligned}
$$

On the other hand, for the transverse tow (weft), it is accepted that the fluid front moves inward perpendicular to the fibers and it is used the mean capillary pressure given in [38] considering an hexagonal array of fibers:

$$
\begin{aligned}
p_{\text {cap }, r} & =(\sigma / R) \cdot \frac{\left[\sin \left(\alpha_{\text {sup }}+\theta\right)-\sin \left(\alpha_{\text {inf }}+\theta\right)\right]}{\left(\frac{5 \pi}{6}(1+\eta)-1-\sqrt{3} / 2\right)} \\
\eta & =d / R=\sqrt{\pi /\left(2 \sqrt{3}\left(1-\varepsilon_{t}\right)\right)}-1 \\
\alpha_{\text {sup }} & \cong(\pi / 2-\theta) \\
\alpha_{\text {inf }} & \cong(\theta-\pi / 2)
\end{aligned}
$$

where $R$ and $d$ are the fiber radius and half-distance between fibers, respectively. The model of Gebart [16] for a hexagonal array is used to calculate the permeabilities in the principal directions:

$$
\begin{aligned}
& K_{1}=8 R^{2} \cdot\left(1-V_{f}\right)^{3} /\left(c \cdot\left(V_{f}\right)^{2}\right) \\
& K_{2}=c_{1}\left(\sqrt{V_{f, \text { max }} / V_{f}}-1\right)^{5 / 2} R^{2}
\end{aligned}
$$

where $V_{f}=1-\varepsilon_{t}$ is the fiber volume fraction, while the parameters $c, c_{1}$ and $V_{f, \max }$ are given by the following expressions:

$$
\begin{aligned}
c & =53 \\
c_{1} & =16 /(9 \pi \sqrt{6}) \\
V_{f, \max } & =\pi /(2 \sqrt{3})
\end{aligned}
$$

\section{Integral equation formulations and numerical techniques}

The Stokes-Brinkman problem of this work is solved using direct BEM and DR-BEM for the channel and tows domains, respectively, with the corresponding matching and boundary conditions presented in Section 2. The integral formulation for the Stokes equation is the following:

$$
\begin{aligned}
c_{i j}(\xi) u_{j}(\xi)= & \int_{S} K_{i j}^{(s)}(\xi, y) u_{j}(y) d S_{y} \\
& -\int_{S} U_{i}^{j(s)}(\xi, y) t_{j}(y) d S_{y}
\end{aligned}
$$

where $c_{i j}=(\alpha / 2 \pi) \delta_{i j}$, with $\alpha$ as the solid angle in the source point, whose value is $\alpha=\pi$ for points located over a smooth contour. For points located inside the domain, $c_{i j}=\delta_{i j}$. In (36), $u_{j}$ and $t_{j}$ are the velocities and tractions in the field points, respectively.

The fundamental solutions of the integral kernels of (36) are given by:

$$
\begin{aligned}
U_{i}^{j(s)}(\xi, y) & =-\frac{1}{4 \pi}\left[\ln \left(\frac{1}{r}\right) \delta_{i j}+\frac{\left(\xi_{i}-y_{i}\right)\left(\xi_{j}-y_{j}\right)}{r^{2}}\right] \\
K_{i j}^{(s)}(\xi, y) & =-\frac{1}{\pi} \frac{\left(\xi_{i}-y_{i}\right)\left(\xi_{j}-y_{j}\right)\left(\xi_{k}-y_{k}\right) n_{k}(y)}{r^{4}} \\
r & =|\xi-y|
\end{aligned}
$$

where $\xi$ and $y$ are the source and field points, respectively.

On the other hand, for the anisotropic Brinkman equation, Khor et al [26] deduced fundamental solutions in the transformed Fourier space, which reduce to the isotropic fundamental solutions in the real space when $\chi_{1}=\chi_{2}$ $[27,62]$. However, the transformation of these functions to 
the real space in the anisotropic case is not a trivial problem and to avoid these difficulties, a boundary-domain integral formulation in terms of the Stokes fundamental solutions is considered for the Brinkman equation and the resulting domain integral is transformed into a boundary integral using DR-BEM [42]. The integral formulation is as follows:

$$
\begin{aligned}
c_{i j}(\xi) u_{j}(\xi)= & \int_{S} K_{i j}(\xi, y) u_{j}(y) d S_{y} \\
& -\int_{S} U_{i}^{j}(\xi, y) t_{j}(y) d S_{y} \\
& +\int_{\Omega} U_{i}^{j}(\xi, y) g_{j}(y) d \Omega_{y}
\end{aligned}
$$

In DR-BEM, the non-homogeneous term, $g_{j}(y)=$ $\chi_{j}^{2} u_{j}(y)$, is approximated using Radial Basis Function (RBF) interpolation given by Augmented Thin Plate Splines (ATPS). The augmented part of a generalized thin plate spline of order $n$ is a polynomial of order $n-1$ that is added to obtain an invertible interpolation matrix [18]. In the present work, $n=2$ and the form of the ATPS is as follows:

$$
\begin{aligned}
& f^{m}(y)=r^{2} \ln (r) \text { for } m=1 \ldots, N_{B}+N_{L} \\
& f^{m}(y)=1 \quad \text { for } m=N_{B}+N_{L}+1 \\
& f^{m}(y)=y_{1} \quad \text { for } m=N_{B}+N_{L}+2 \\
& f^{m}(y)=y_{2} \quad \text { for } m=N_{B}+N_{L}+3
\end{aligned}
$$

where $N_{B}$ is the number of boundary points, $N_{L}$ is the number of interior points and $r\left(y, z^{m}\right)=\left|y-z^{m}\right|$ is the distance between the field points, $y$, and the trial points, $z^{m}$. Accordingly, the non-homogeneous term can be expanded as follows:

$$
g_{j}(y)=\sum_{m=1}^{N_{B}+N_{L}+3} \alpha_{l}^{m} \delta_{j l} f^{m}(y), \quad j=1,2 ; l=1,2
$$

where $\alpha_{l}^{m}$ represent the approximation coefficients in the direction $l$. The ATPS represented in (41) to (44) requires the addition of orthogonality conditions, as shown in the following equation:

$$
\sum_{m=1}^{N_{B}+N_{L}} \alpha_{l}^{m}=\sum_{m=1}^{N_{B}+N_{L}} \alpha_{l}^{m} y_{1}^{m}=\sum_{m=1}^{N_{B}+N_{L}} \alpha_{l}^{m} y_{2}^{m}=0
$$

After substituting (45) into (40), the integral representation takes the following form:

$$
\begin{aligned}
c_{i j}(\xi) u_{j}(\xi)= & \int_{S} K_{i j}(\xi, y) u_{j}(y) d S_{y} \\
& -\int_{S} U_{i}^{j}(\xi, y) t_{j}(y) d S_{y} \\
& +\sum_{m=1}^{N_{B}+N_{L}+3} \alpha_{l}^{m} \int_{\Omega} U_{i}^{j}(\xi, y) \delta_{j l} f^{m}(y) d \Omega_{y}
\end{aligned}
$$

The transformation of the domain integral into a boundary integral is accomplished by defining the following auxiliary Stokes field:

$$
\begin{aligned}
& \frac{\partial \hat{u}_{j}^{(m l)}}{\partial y_{j}}=0 \\
& \mu \frac{\partial^{2} \hat{u}_{j}^{(m l)}}{\partial y_{k} \partial y_{k}}-\frac{\partial \hat{p}^{(m l)}}{\partial y_{j}}=f^{m}(y) \delta_{j l}
\end{aligned}
$$

with the particular solutions for the ATPS given in [10].

The substitution of the auxiliary field defined in (48) and (49) into (47) and the application of the Green's identities in the domain integral, lead to the following boundary-only integral representation:

$$
\begin{aligned}
c_{i j}(\xi) u_{j}(\xi)= & \int_{S} K_{i j}(\xi, y) u_{j}(y) d S_{y} \\
& -\int_{S} U_{i}^{j}(\xi, y) t_{j}(y) d S_{y} \\
& +\sum_{m=1}^{N_{B}+N_{L}+3} \alpha_{l}^{m}\left(c_{i j}(\xi) \hat{u}_{j}^{(m l)}(\xi)\right. \\
& -\int_{S} K_{i j}(\xi, y) \hat{u}_{j}^{(m l)}(y) d S_{y} \\
& \left.+\int_{S} U_{i}^{j}(\xi, y) \hat{t}_{j}^{(m l)}(y) d S_{y}\right)
\end{aligned}
$$

where the coefficients $\alpha_{l}^{m}$ are given in terms of the inverse of the interpolating matrix obtained by collocation of (45) at $N_{B}$ boundary nodes and $N_{L}$ internal nodes.

The singularities arising in the kernels $K_{i j}$ and $U_{i}^{j}$ of (36) and (50) are dealt with the rigid body motion principle [45] and the Telles transformation [61], respectively. In these equations, the boundary and the physical variables are discretized using quadratic isoparametric interpolation. Additionally, discontinuous shape functions with a collocation factor of $\alpha_{d i s}=2 / 3$ are employed at the corners of the problem domain [69]. After the discretization of the contour 
and variables and the assembly of the resultant matrices, by considering the corresponding boundary and matching conditions, a linear system of equations is obtained, which is solved using singular value decomposition (SVD) to consider cases where the condition number of the global matrix is very large due to large differences on the magnitude of the problem parameters. In the DR-BEM formulation the coordinate systems of the saturated porous domains are continuously updated as the fluid front advances, in such a way that each coordinate system is located in the corresponding centroid of each saturated domain to avoid the increment of the condition number of the final system as the filling takes place.

First order Euler integration of the kinematic condition (18) is used to track the moving fronts. As an explicit time stepping-algorithm is used, the time step needs to be restricted to small values. The Courant-Friedrich-Levy (CFL) condition is used to guarantee the stability of the solution as time progresses, with the CFL constant changing as a function of the capillary ratio, $C_{\text {cap }}$. As $C_{c a p}$ increases, smaller values of this constant are required, leading to shorter time intervals. Additional geometrical restrictions shall be considered to compute the time interval in order to avoid the crossing of the evolution points as the fluid front advances and to avoid the advancement of the points beyond the limits between the two regions considered (channels and bundles) or beyond the boundaries of the global domain. At a point $x^{i}$ on the moving fluid front, both the unit normal vector, $\hat{n}^{i}$, and the curvature, $\kappa^{i}$, are computed numerically using the following fourth-order lagrangian polynomial [52]:

$$
\begin{aligned}
\left(x_{j}^{\prime}\right)^{i} & =1 / 6\left(x_{j}^{i-2}-8 x_{j}^{i-1}+8 x_{j}^{i+1}-x_{j}^{i+2}\right), j=1,2 \\
\left(x_{j}^{\prime \prime}\right)^{i} & =\frac{\left(-x_{j}^{i-2}+16 x_{j}^{i-1}-30 x_{j}^{i}+16 x_{j}^{i+1}-x_{j}^{i+2}\right)}{3} \\
\hat{n}^{i} & =\frac{1,2}{\left(\sqrt{\left(\left(x_{1}^{\prime}\right)^{i}\right)^{2}+\left(\left(x_{2}^{\prime}\right)^{i}\right)^{2}}\right)} \cdot\left(\left(x_{2}^{\prime}\right)^{i},-\left(x_{1}^{\prime}\right)^{i}\right) \\
\kappa^{i} & =\frac{\left[\left(x_{1}^{\prime}\right)^{i}\left(x_{2}^{\prime \prime}\right)^{i}-\left(x_{2}^{\prime}\right)^{i}\left(x_{1}^{\prime \prime}\right)^{i}\right]}{\left[\left(\left(x_{1}^{\prime}\right)^{i}\right)^{2}+\left(\left(x_{2}^{\prime}\right)^{i}\right)^{2}\right]^{3 / 2}}
\end{aligned}
$$

Once the meshes of the moving fronts have been reconstructed at the current time step and the normal and curvatures have been computed, the BEM/DR-BEM algorithm is used to calculate the velocity of the moving fronts and the cycle is repeated again in a quasi-static approach given the low
Reynolds number of the problem. A more detailed description of the tracking technique of the fluid front can be found in a work recently published [43].

\section{Results and discussion}

\subsection{Assessment of accuracy and convergence}

To validate the proposed BEM/DR-BEM scheme for the solution of coupled Stokes-Brinkman problems a fully developed flow in two horizontal layers, porous medium and channel, is considered as shown in Fig. 1. This problem admits analytical solution and has been used by other authors to validate FEM codes $[11,23,58]$. In this problem, the inlet and outlet boundaries are subjected to pressure boundary conditions and the upper and lower ones, to symmetry boundary conditions. Both continuous and jump stress matching conditions at the interface between the two layers are considered. Equations (55) and (56) give the analytical solution for the velocity profiles (with $\phi=\mu / \mu_{e f f}$ ), which is only valid provided that the boundary layer thickness of the Brinkman flow is smaller than the height of the porous medium, in such a way that the solution tends to a Darcy flow in the lower part of the porous medium domain.

$$
\begin{aligned}
\widehat{\mathrm{u}_{x}^{s}}(\hat{y})= & -(\hat{H} / 2) \cdot\left(\hat{y}-\hat{y}^{2} / \hat{H}\right) \cdot(\Delta \hat{p} / \Delta \hat{x})^{s} \\
& -\left(\hat{H} /\left(2 L^{*}\right)\right) \sqrt{\phi K_{1}} /(1+\beta \sqrt{\phi})(\Delta \hat{p} / \Delta \hat{x})^{s} \\
& -K_{1} /\left(L^{* 2}(1+\beta \sqrt{\phi}) \phi\right)(\Delta \hat{p} / \Delta \hat{x})^{b} \\
\widehat{\mathrm{u}_{x}^{b}}(\hat{y})= & -K_{1} /\left(L^{* 2} \phi\right)(\Delta \hat{p} / \Delta \hat{x})^{b} \\
& -\frac{\left[\left(\hat{H} /\left(2 L^{*}\right)\right) \sqrt{\phi K_{1}}(\Delta \hat{p} / \Delta \hat{x})^{s}+K_{1} /\left(L^{* 2} \phi\right)(\Delta \hat{p} / \Delta \hat{x})^{b}\right]}{(1+\beta \sqrt{\phi})} \\
& \times e^{\left(\sqrt{\varphi / K_{1}} \hat{y} L^{*}\right)}+K_{1} /\left(L^{* 2} \phi\right)(\Delta \hat{p} / \Delta \hat{x})^{b} e^{\left(\sqrt{\varphi / K_{1}} \hat{y} L^{*}\right)}
\end{aligned}
$$

This problem was solved by [23] using a modified Brinkman approach for the whole domain that reduces to the Stokes flow in the channel domain. In this approach, the stress jump condition is incorporated by a level-set formulation

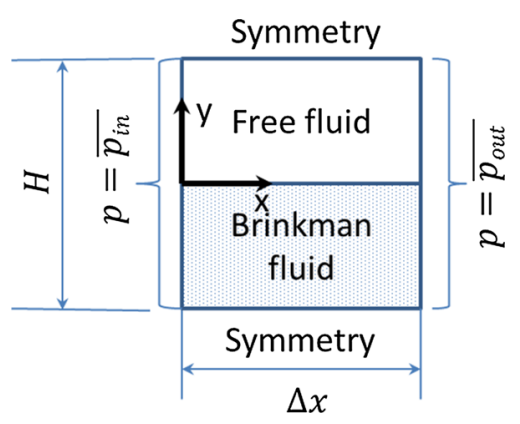

Fig. 1 Scheme of coupled problem Stokes-Brinkman admitting analytical solution 
and the numerical solution is obtained by the finite element method (FEM). The authors considered the following parameters in the simulations: $H=1 \mathrm{~cm}, \beta=0.7, \quad \mu_{\text {eff }}=\mu / \varepsilon_{t}$ with $\varepsilon_{t}=0.5$, and $K=10^{-4} \mathrm{~cm}^{2}$, where $K$ is the permeability of the porous medium in the flow direction. Considering a characteristic length of $L^{*}=H$ for this problem, the inverse Darcian number is $\chi^{2}=5.00 \times 10^{3}$. Four mesh-sizes were evaluated, $h=0.1 \mathrm{~cm}, h=0.05 \mathrm{~cm}, h=0.01 \mathrm{~cm}$ and $h=0.005 \mathrm{~cm}$, using a uniform distribution of a regular mesh of square elements over the entire domain, with $h$ as the length of the side of one square element, obtaining a total number of elements in each case equal to $0.1 / h^{2}$. In a similar fashion, in the present case, $h$ represents the size of one quadratic element of the contour mesh, which leads to $N_{E}=(3 \times 0.1+2 \times 1) / h, N_{B}=4(0.6 / h+1)$ and $N_{L}=(0.2 / h-1) \times(1 / h-1)$, with $N_{E}$ as the number of boundary elements of the whole domain (Stokes and Brinkman), whereas $N_{B}$ and $N_{L}$ are the boundary and interior DR-BEM trial points in the Brinkman domain, respectively. The characteristics of the meshes for this particular case and the corresponding $\mathrm{L}^{2}$ relative error norms of the BEM/DRBEM solution are presented in Table 1, as well as the convergence rate, $n_{c}$. The $\mathrm{L}^{2}$ relative error norm is defined as:

$L^{2}=\left(\frac{\sum_{i=1}^{N_{B}+N_{L}}\left(\widehat{u_{x} \text { anal }}-\widehat{u_{x} \text { num }}\right)^{2}}{\sum_{i=1}^{N_{B}+N_{L}}\left(\widehat{u_{x} \text { anal }}\right)^{2}}\right)^{1 / 2}$,

where $\widehat{u_{x} \text { anal }}$ and $\widehat{u_{x} \text { num }}$ are the analytical and numerical solutions of the dimensionless horizontal velocity, respectively. The convergence rate, $n_{c}$, corresponds to the exponent of the power curve that fits to the data of $L^{2}$ relative error norm versus Element Size, i.e., $L^{2}=a \cdot h^{n_{c}}$, where $a$ is a constant and $n_{c}$ is the slope in a $\log -\log$ plot of the $L^{2}$ relative error versus Element Size (see Fig. 2 with the corresponding coefficient of determination $R^{2}=0.988$ ).

According to [3], the application of the Finite Element Method (FEM) to fluid flow problems implies the use of mixed formulations where multiple field variables shall be considered, like the velocity and pressure in the case of an incompressible fluid flow. In such a cases, the discretization scheme of the domain should fulfill three conditions with the purpose to assure the solvability, stability and optimality of the FEM solution, namely, consistency, ellipticity and infsup condition, being the last one the most difficult to satisfy due to the choice of numerical constants to be introduced in the formulation or to the modification of the original FEM scheme in order to satisfy implicitly such a condition. The statement of the Inf-Sup condition depends on the problem being analyzed; in the case of Stokes-Darcy problems, this condition is detailed in [36]. For both Stokes and Brinkman flows, some distorted FEM meshes could not satisfy the Inf- 


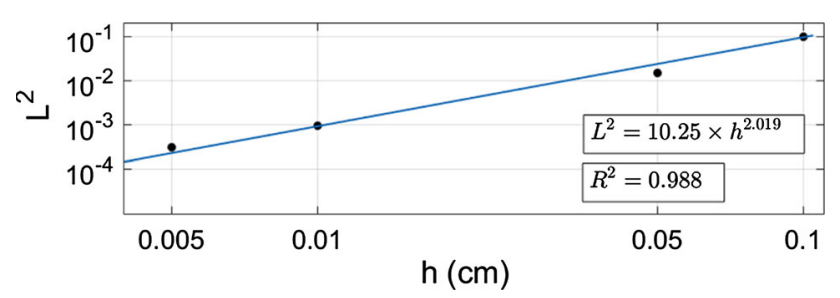

Fig. 2 Plot of convergence of the BEM/DR-BEM solution

Sup condition, resulting in spurious pressure modes (for more details see [3]). On the other hand, in the BEM/DR-BEM formulation for both flow fields, spurious pressure modes are not possible due to the unique relationship between the velocity and pressure fundamental solutions in the integral formulation of the problems. However, significant numerical errors and inaccuracy can be found by using very distorted BEM meshes, due to ill conditioning of the global matrix system and/or near singularities in the numerical integrations. Therefore, in our scheme, it is convenient to avoid a distorted mesh, i.e., a mesh having adjacent elements with very dissimilar sizes, to preserve the accuracy of the solution. As mentioned before, since the points at the fluid front are not uniformly spaced just after the fluid front advancement, we have implemented a remeshing algorithm with the purpose to obtain a balanced mesh in every time instant and avoid in this way the loss of accuracy in the solution.

The comparison of the present BEM/DR-BEM scheme with the FEM scheme of [23] is shown in Fig. 3a, b, where a semi-log plot is adopted in order to distinguish the velocities in the porous domain, which can be several orders of magnitude lower than the velocities in the channel. In the Brinkman domain, the positions of the interior trial points of the RBF interpolation are determined by an extension into the domain of the boundary mesh points, with exception of the points close to the corners. In both schemes, BEM/DR-BEM and FEM, the numerical solution converges to the analytical one, reaching a high order of accuracy when using the finer mesh, which corresponds to a value of $h=0.005 \mathrm{~cm}$, i.e., 4.000 square finite elements uniformly distributed for the FEM scheme, and, in the case of the BEM/DR-BEM scheme, 460 boundary elements distributed over the external boundary and interface between the two domains (Stokes and Brinkman), with 484 boundary and 7761 interior interpolation points in the porous domain (Brinkman), as shown in Table 1.

Two main differences between the two results can be observed. Firstly, the BEM/DR-BEM shows high accuracy in the Stokes velocity profile, channel flow, for all mesh sizes, while the Brinkman velocity profile is over-predicted for the coarser meshes (Fig. 3a). On the other hand, the FEM scheme always predicts an accurate velocity profile in the porous medium (Brinkman), but the velocity profile in the
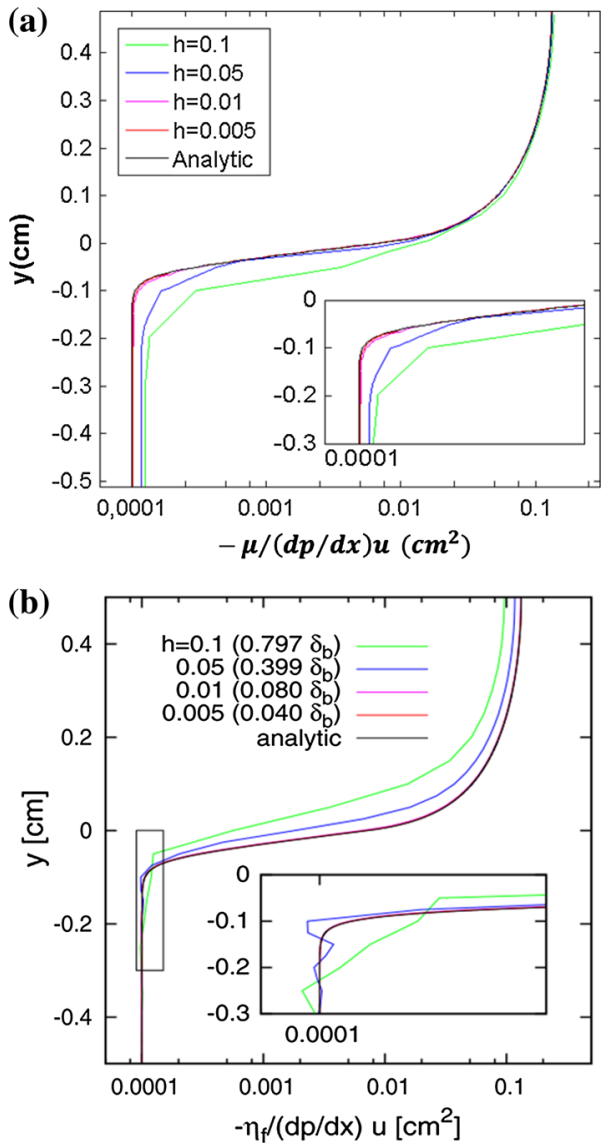

Fig. 3 Velocity profiles for coupled Stokes-Brinkman problem. a BEM/DR-BEM approach. b FEM approach

channel (Stokes) is under-predicted for the coarser meshes (Fig. 3b). As commented in [23] the observed behavior in the FEM solution is due to the interpolation scheme used in the level-set formulation. To be more specific, it is necessary to mention that a single equivalent momentum equation for the coupled Stokes-Brinkman domain was considered in [23], which is essentially a Stokes equation modified with permeability and jump stress terms to account for the fluid flow in the porous medium and the stress matching condition in the interface, respectively. Additionally, the domain geometry was defined by a level set function, $\Phi$, where $\Phi=0$ at the interface, $\Phi>0$ at the free-fluid domain and $\Phi<0$ at the porous medium. When $|\Phi|<\varepsilon_{\text {int }}$, with $\varepsilon_{\text {int }}$ as the half thickness of the diffuse interfacial region, an interpolation function is defined to express the permeability and jump stress terms as a function of $\Phi$ and $\varepsilon_{i n t}$, and the errors of such interpolation led to greater numerical errors in the Stokes velocity profile, as explained in [23]. On the other hand, the observed behavior of the numerical solution with the mesh size in the BEM/DR-BEM approach is due to the approximation error on the evaluation of the volume integral in the integral representation formula (40) used to represent the Brinkman 
flow. As mentioned before, in the case of the Stokes flow an exact only-boundary integral formulation is known, equation (36), requiring only the discretization of the boundary integral densities, $u_{i}$ and $t_{i}$, without any additional approximation. The discretization of the densities, $u_{i}$ and $t_{i}$, is also required in (40), as well as the corresponding approximation of the volume integral. The transformation of the domain integral appearing in (40) into the boundary integrals arising in (50) by DR-BEM approximation involves the interpolation of the non-homogeneous term, $g_{j}(y)$, using Augmented Thin Plate Splines (ATPS) as shown in (45). In this case, the approximation error of such interpolation, which is greater as the permeability is lower and/or the mesh is coarser, is the principal error source of the Brinkman velocity profiles. It is worth-mentioning that in the BEM /DR-BEM results no oscillations are present in points close to the interface Stokes-Brinkman for any mesh-size, contrary to the FEM scheme used in [23] where oscillations can be noticed for the coarser meshes (see details of Fig. 3a, b).

To analyze the influence of the non-dimensional parameters $\chi^{2}$ and $\beta$ on the accuracy of the solution, several simulations were performed using the data summarized in Table 2. As observed, simulations for values of $\chi^{2}=1 \times$ $10^{4}, \quad \chi^{2}=1 \times 10^{3}, \chi^{2}=1 \times 10^{2}$ and $\chi^{2}=18$ were carried out, considering in each case three different values of the jump stress coefficient, namely, $\beta=0, \quad \beta=0.5$, and $\beta=1.0$. In all cases, the same number of boundary elements and interpolation trial points were considered, namely, 49 boundary elements, 56 boundary and 95 interior interpolation points in the porous domain (Brinkman). In Table 2 the obtained $\mathrm{L}^{2}$ relative error norm for each case is also reported. As observed, the accuracy of the solution improves as the value of $\beta$ increases for $\chi^{2}=1 \times 10^{4}, \quad \chi^{2}=1 \times 10^{3}$ and $\chi^{2}=1 \times 10^{2}$, however, for the case of $\chi^{2}=18$, this behavior is reversed. Besides, for a given value of $\beta$, a higher accuracy is found as $\chi^{2}$ decreases, which is reasonable because in the BEM/DR-BEM scheme the approximation error of the nonhomogeneous term of the Brinkman equation has a stronger influence on the results as $\chi^{2}$ is larger.

The velocity profiles corresponding to the simulations of Table 2 and their comparison with the analytical solutions given by (55) and (56) are presented in Fig. 4a-d. The magnitude of the Stokes velocity increases as the value of $\beta$ reduces, being this effect more significant as $\chi^{2}$ is lower (see Fig. 4d). As expected, for a constant $\beta$, the thickness of the boundary layer increases with the reduction of $\chi^{2}$. On the other hand, for a constant value of $\chi^{2}$, the boundary layer thickness increases as the value of $\beta$ reduces, being this change more notorious as $\chi^{2}$ is smaller. Similar results to those ones shown in Fig. 4a-d were reported in [58] using a FEM approach, which means that the present scheme is consistent with previously reported results.

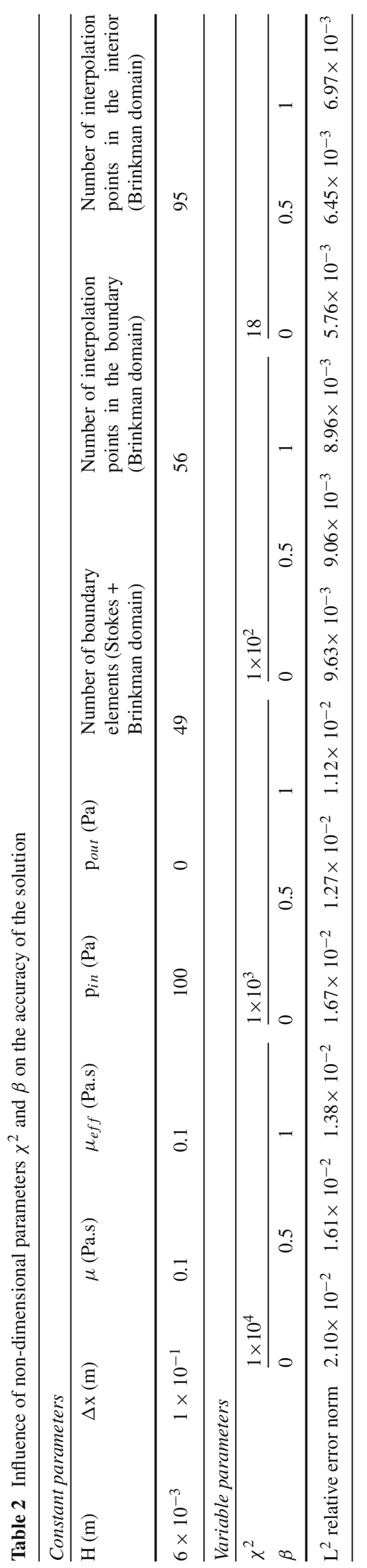



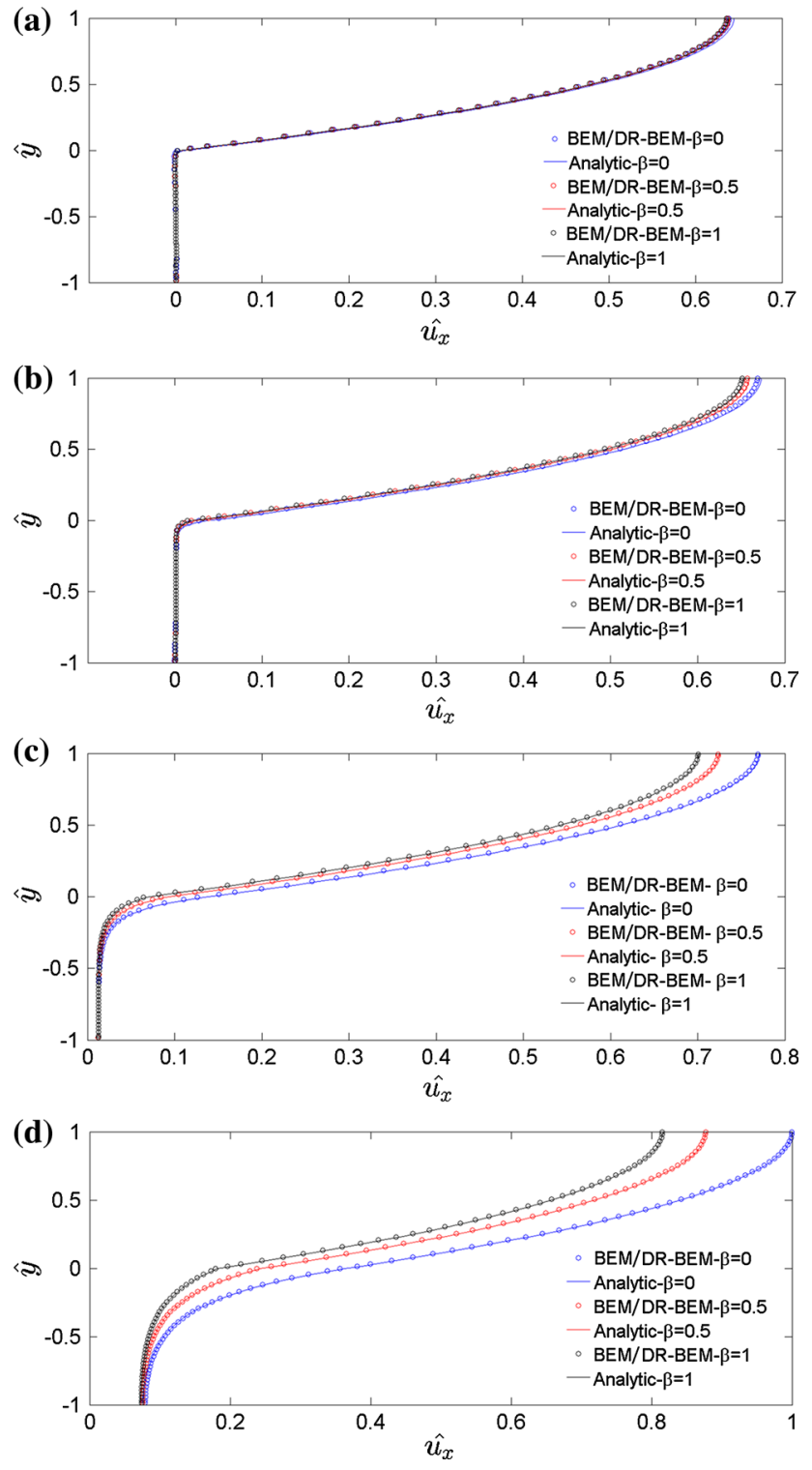

Fig. 4 Graphical comparison between analytical and numerical solutions for coupled Stokes-Brinkman problem. a $\chi^{2}=1 \times 10^{4}$, b $\chi^{2}=1 \times 10^{3}, \mathbf{c} \chi^{2}=1 \times 10^{2}, \mathbf{d} \chi^{2}=18$

\subsection{Statement of problem of void formation, simulation data and void characterization}

In the processing of composites materials, different sorts of RUC architectures can be examined for dual-scale fibrous reinforcements. In this section, it is considered a 2D geometry emulating a longitudinal plane of a cross ply fabric (Fig. 5). The unidirectional filling of 3D RUC's of fabrics has been recently considered by superposition of $2 \mathrm{D}$ simulations at several longitudinal planes of the RUC, showing good agreement with experimental results [7]. Therefore, we can infer that the parametric study for the 2D geometry represented in the Fig. 5 can be useful to understand the influence of some factors on the void formation in cross ply fabrics.

Simulations considered in the following sections are classified into four different series. Series 1 to 3 provide different data to be used in the simulations performed with the StokesBrinkman formulation, while Serie 4 defines the data of the simulations run with the Stokes-Darcy formulation. The parameters of the Serie 1, which is taken as the reference case, are presented in Table 3, where the fixed parameters are prescribed and the calculated parameters are determined from the former ones. The jump stress coefficient used in the simulations of Serie $1, \beta=1.24$, was calculated using the model of Valdés-Parada et al. [64,65] together with the Larson-Hidgon coefficient in the channel-tow interface, $\gamma^{*}$ [29] :

$$
\begin{aligned}
\beta & =\frac{2 \varepsilon_{c t}^{3 / 2}}{3 \sqrt{\gamma^{*}\left(1+\varepsilon_{c t}\right)^{3}}} \\
\gamma^{*} & =\left(2 \times 10^{-3}\right) /\left(1-\varepsilon_{c t}\right)^{2 / 3}
\end{aligned}
$$

where the porosity of the channel-tow interface, $\varepsilon_{c t}$, is approximated as the porosity of the bundle, $\varepsilon_{t}$, and $\mu_{e f f}=$ $\mu / \varepsilon_{t}$. In Serie 2, the jump stress coefficient is changed to $\beta=0.7$, corresponding to the approximation considered in [23], and $\mu_{e f f}=\mu / \varepsilon_{t}$ as before. For Serie 3, the continuous stress condition is considered, i.e. $\beta=0$, and $\mu_{\text {eff }}=\mu$.
Fig. 5 Scheme of problem of simultaneous filling of a RUC

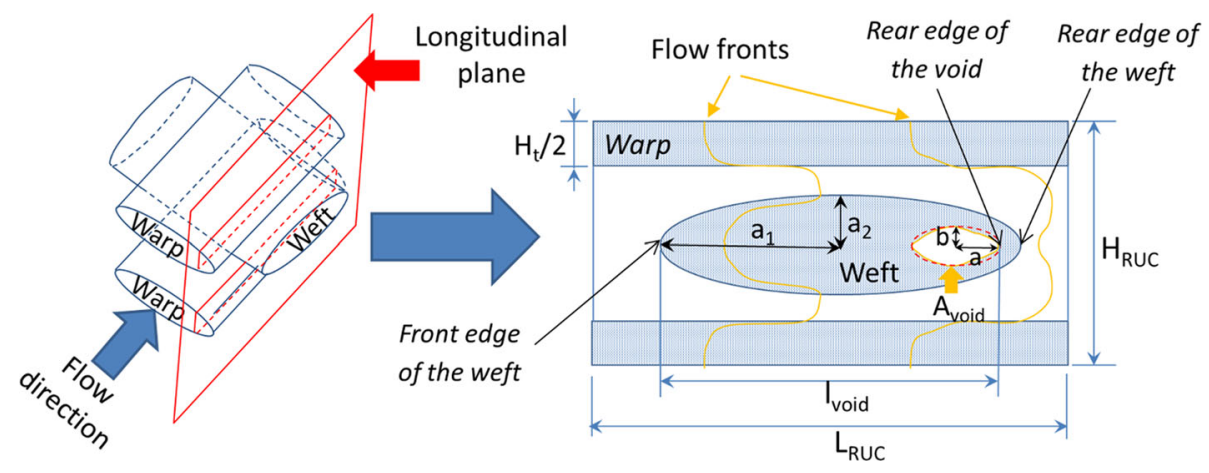


For the jump stress cases (Serie 1 and 2), considering a characteristic length of $L^{*}=H_{t}$, where $H_{t}$ is the tow height (see Fig. 5), the inverse Darcian numbers in the principal directions are $\chi_{1}^{2}=1.40 \times 10^{3}$ and $\chi_{2}^{2}=6.80 \times 10^{3}$, whereas for the continuous stress case (Serie 3 ), the corresponding values are $\chi_{1}^{2}=2.26 \times 10^{3}$ and $\chi_{2}^{2}=1.10 \times 10^{4}$. For simulations of Serie 4 , a Stokes-Darcy formulation is considered and therefore the stress jump condition is not applicable. For this type of formulation, uniqueness of the solution requires continuity of surface tractions and a jump condition for the interfacial tangential velocities given by the Beavers-Joseph slip condition, for more details see (71) and (72). The slip coefficient, $\gamma$, of the slip condition, see (71), is usually found by mean of experiments, but when the ratio between the height of the porous medium and the square root of the permeability is very high, a good approximation for this coefficient is $\gamma=1 /\left(\varepsilon_{t}^{1 / 2}\right)$ [22]. The formulation is completed by requiring continuity of the normal component of the interfacial velocities, see (70). In Section 4.3, the results obtained using the Stokes-Darcy formulation are compared with those ones obtained with the Stokes-Brinkman formulation.

As a constant inlet pressure condition is considered in all simulations, the comparison between the pressure and capillary forces is described by the capillary ratio, $C_{\text {cap }}=$ $P_{c a p, \max } / P_{i n}$, as defined in [30], where $P_{c a p, \max }$ and $P_{i n}$ stand for the maximum capillary pressure and inlet pressure, respectively. For Series 1, 2 and 4, three different capillary ratios are contemplated: $C_{\text {cap }}=1 \times 10^{-2}, C_{\text {cap }}=1 \times 10^{-1}$ and $C_{c a p}=5 \times 10^{-1}$, whereas for Serie 3 only the lower value of $C_{c a p}$ is taking into account, i.e, $C_{\text {cap }}=1 \times 10^{-2}$. For each simulation the following parameters are achieved:

- Size, shape and location of voids, expressed as (see Fig. 5):

$$
\begin{aligned}
\text { Size } & =A_{\text {void }} /\left(2 A_{R U C}\right) \\
\text { Shape } & =a / b \\
\text { Location } & =l_{\text {void }} /\left(2 a_{1}\right)
\end{aligned}
$$

where $A_{\text {void }}$ is the void area, $A_{R U C}$ is the RUC area, $a$ and $b$ are the semi-major and semi-minor axes of the ellipse circumscribing the void, $a_{1}$ is the semi-major axis of the weft and $l_{\text {void }}$ is the distance between the front edge of the weft and rear edge of the void.

- Dimensionless time, $\hat{t}$, defined by (6), and normalized time that is defined as $t^{*}=t / t_{\text {filling }}$, in which $t_{\text {filling }}$ is the total filling time of the RUC. In this work, two adjacent RUC's are considered and the filling stops when the partial equilibrium of the bubble formed in the second RUC has been reached.

- RUC's saturation versus Normalized time 
- Compression of the voids, defined by the following compressibility ratio:

$$
\psi=\frac{V_{\text {void }}^{o}-V_{\text {void }}^{f}}{V_{\text {void }}^{f}}
$$

where $V_{\text {void }}^{o}$ and $V_{\text {void }}^{f}$ stand for the initial and final volume of the void, respectively.

The simulations of the Sects. 4.3 and 4.4 are performed with the same mesh-size used in the simulations of Fig. 4. The number of boundary elements and interpolation points changes continuously as the fluid front evolves.

\subsection{Comparison between Stokes-Darcy and Stokes-Brinkman approaches}

\subsubsection{Description of the Stokes-Darcy approach}

Two coupled domains shall be considered in the mesoscopic modeling of dual-scale fibrous reinforcements, namely, channels and tows. The modeling of the flow in the porous media (tows) can be done by the Brinkman equation, which reduces to the Darcy equation when the permeability is very low. Despite that the majority of cases of composite processing involve low permeability tows and, therefore, are prone to be modeled by the Darcy approximation, some authors prefer to use the Brinkman equation in its original form $[11,23,58]$. This is due to the possibility of imposing explicitly the matching conditions at the channel-tow interface in terms of the interfacial velocity and surface traction components, given that the Brinkman and Stokes partial differential equations are of the same order. As expected, several authors have used the Darcy equation to model the flow in the tows [24,43,60], but, considering that this is a first order partial differential equation, a jump matching condition on the tangential velocities at the channel-tow interface is required, which is linear proportional to the Stokes surface traction in the channel and is function of a slip coefficient, $\gamma$ (Beavers-Joseph slip condition). The value of the slip coefficient, $\gamma$, appearing in this condition is still matter of controversy. Considering that both approaches, Stokes-Brinkman and Stokes-Darcy, have been used in the literature and are consistent with the problem dealt here, the present section is devoted to compare the results of void formation obtained by both of them, namely, Serie 1 for Stokes-Brinkman and Serie 4 for Stokes-Darcy. It is important to mention that a coupled solution system is considered for both approaches, i.e., the equations of the freefluid and porous medium domains, as well as the matching conditions, are directly included in a single solution system, which could be ill-conditioned as mentioned before. In other works, decoupling strategies have been used for the solu- tion of Stokes-Darcy problems, such as: iterative subdomain methods [8], Lagrange multipliers [31], two-grid method [37], among others. For instance, Mu and Zhu [36], who used the Saffman matching condition for the tangential velocities (which comes from disregarding the tangential Darcy velocity in the Beavers-Joseph condition), proposed and assessed a decoupling FEM methodology based on interface approximations via temporal extrapolation for non stationary cases. This methodology allows solving two decoupled subproblems independently by invoking conventional Stokes and Darcy solvers. After analyzing the behavior of the convergence rate and approximation errors with the time step and element size regarding a coupled strategy, it was concluded that the proposed methodology is computationally effective for this kind of problems.

In dimensionless form, the Darcy equation is as follows:

$\hat{u}_{i}=-K_{i}^{*}\left(\frac{\partial \hat{p}}{\partial \hat{x}_{i}}\right)$

where $K_{i}^{*}=K_{i} /\left(L^{*}\right)^{2}$ is the non-dimensional permeability in the principal direction $i, \hat{p}=p /\left(\mu . U_{\max } / L^{*}\right)$ is the nondimensional pressure, $\widehat{x_{i}}=x_{i} / L^{*}$ and $\hat{u}_{i}=u_{i} / U_{\max }$ are the non-dimensional coordinate and non-dimensional velocity in direction $i$, respectively. The integral representation formula for the pressure field when the Darcy velocity (64) satisfies mass conservation (1) is given as (for more details see $[6,13$, 44]):

$c(\xi) p(\xi)=\int_{S} p^{*}(\xi, y) q(y) d S_{y}-\int_{S} q^{*}(\xi, y) p(y) d S_{y}$

where:

$$
\begin{aligned}
p^{*}(\xi, y) & =-\frac{1}{2 \pi} \ln \left(r_{e}\right) \\
q^{*}(\xi, y) & =\frac{K_{i}}{K_{e}} \frac{\partial p^{*}}{\partial y_{i}}(\xi, y) \widehat{n_{i}}(y) \\
& =-\frac{\left[\left(y_{1}-\xi_{1}\right) \widehat{n_{1}}(y)+\left(y_{2}-\xi_{2}\right) \widehat{n_{2}}(y)\right]}{2 \pi\left(r_{e}\right)^{2}} \\
r_{e} & =\left[\left(\frac{K_{2}}{K_{1}}\right)^{\frac{1}{2}}\left(y_{1}-\xi_{1}\right)^{2}+\left(\frac{K_{1}}{K_{2}}\right)^{\frac{1}{2}}\left(y_{2}-\xi_{2}\right)^{2}\right]^{1 / 2} \\
K_{e} & =\left(K_{1} K_{2}\right)^{1 / 2}
\end{aligned}
$$

At the channel-tow interfaces, the flow fields defined by (36) and the corresponding pressure (Stokes flow), and (64) and (65) (Darcy flow), have to satisfy the following matching conditions: 
- Continuity of normal velocities:

$$
\hat{u}_{i}^{(s)} \hat{n}_{i}=\hat{u}_{i}^{(d)} \hat{n}_{i}
$$

- Beavers-Joseph slip condition for tangential velocities:

$$
\left(\frac{\partial \hat{u}_{i}}{\partial \hat{x}_{j}}+\frac{\partial \hat{u}_{j}}{\partial \hat{x}_{i}}\right)^{(s)} \hat{n}_{j} \widehat{\tau}_{i}=\frac{\gamma L^{*}\left(\hat{u}_{i}^{(d)}-\hat{u}_{i}^{(s)}\right) \widehat{\tau}_{i}}{\sqrt{\left(K_{1}+K_{2}\right) / 2}}
$$

- Continuity of surface tractions:

$$
\hat{n}_{i} \hat{\sigma}_{i j}^{(s)} \hat{n}_{j}=-\hat{p}^{(d)}
$$

where $s$ stands for Stokes and $d$ stands for Darcy. Here $\hat{n}_{i}$ is the normal vector outwardly oriented from the Stokes domain, $\gamma$ is the slip coefficient, $\widehat{\tau_{i}}$ is the tangential vector, $K_{1}$ and $K_{2}$ are the main permeabilities, $\hat{\sigma}_{i j}^{(s)}$ is the dimensionless Cauchy stress tensor in the Stokes domain and $\hat{p}^{(d)}$ is the dimensionless pressure in the Darcy domain. In the BeaversJoseph condition (71), the tangential Darcy velocity cannot be disregarded a priori and is approximated in terms of the interface Darcy pressure using Lagrange interpolation functions for quadratic elements, $\mathrm{L}_{j}(\zeta)$, as follows (Sum on $i$ and $j$ ):

$$
u_{i}^{(d)} \widehat{\tau_{i}}=-\left(K_{i} / \mu\right)\left(\partial \zeta / \partial x_{i}\right) \widehat{\tau}_{i}\left(\partial \mathrm{L}_{j}(\zeta) / \partial \zeta\right) p_{j}
$$

with $\zeta \in[-1,1]$.

At the inlet of the Darcy domain, the non-dimensional boundary condition is:

$\hat{p}^{(d)}=\bar{p} L^{*} /\left(\mu U_{\max }\right)$

while, at the fluid front, the kinematic condition is given by (18) and the dynamic condition is given by:

$\hat{p}^{(d)}=L^{*}\left(p_{a}-p_{\text {cap }}\right) /\left(\mu U_{\max }\right)$

where the parameters appearing in (74) and (75) were previously defined in Section 2. The Stokes-Darcy formulation used in this work was previously validated in [43].

\subsubsection{Comparison of the RUC filling process for $C_{\text {cap }}=1 \times 10^{-2}$}

In Fig. 6, different instants of the filling process predicted by the Stokes-Darcy (S-D) and Stokes-Brinkman (S-B) approaches are compared; in each instant, the fluid front position along the channel is the same for both approaches, S-D and $\mathrm{S}-\mathrm{B}$, but different evolution times are predicted. The fluid front shapes at the first instant look very similar for both approaches (Fig. 6a vs. b), with S-D predicting a slightly greater arrival time than $\mathrm{S}-\mathrm{B}$. As expected, due to the low value of the capillary ratio considered, $C_{c a p}=1 \times 10^{-2}$, the fluid fronts in the channel surpass the fluid front in the tow, and, after a while, the liquid completely surrounds the first weft, see Fig. 6e, f. At the instant corresponding to Fig. 6c, $\mathrm{d}$, when the liquid at the channel is approximately half the way along the first weft, $6.57 \%$ of the total filling time has elapsed according to $\mathrm{S}-\mathrm{D}$, while $\mathrm{S}-\mathrm{B}$ predicts a value of $5.78 \%$. Additionally, the minimum position of the fluid front in both the warps and the weft is barely larger for the S-D simulation. When the channel fluid fronts totally surround the first weft and merge one another, the air is trapped and the void compression starts. According to Fig. 6e, f, the fluid fronts in the warps and the weft for S-D are ahead with respect to $\mathrm{S}-\mathrm{B}$, resulting in a smaller initial void (bubble) in the case of S-D. Moreover, the real arrival time to this position is longer for $\mathrm{S}-\mathrm{B}$, but the normalized time is shorter instead. This last feature is common for all the cases analyzed in Fig. 6a-h, where the normalized times of the fluid front evolution are always shorter for the S-B simulation. Figure $6 \mathrm{~g}$, $\mathrm{h}$ corresponds to the case when the fluid front in the channel is approximately at $80 \%$ of the total length of the domain; at this point of the simulation, the time in the S-D approach is $57.2 \%$ of the total evolution time, while the time in $\mathrm{S}-\mathrm{B}$ is $\mathbf{5 4 . 7 \%}$. This is a manifestation of the reduction of the saturation rate as the fluid front progresses with respect to the initial rate, as it is confirmed later. When the channel fluid front totally surrounds the second weft another bubble is formed, undergoing a compression process until the partial equilibrium is attained (see Fig. 6i, j), as in the first bubble. In these last two figures, it can be observed that the voids predicted by the S-D simulation are smaller than those ones predicted by $\mathrm{S}-\mathrm{B}$, with the bubble of the first weft smaller than the one of the second weft for both approaches. In general, the influence of the type of approach, S-D or S-B, on the void location is not as significant as the influence on the void size and shape, for more details see Table 4

\subsubsection{Effect of the capillary ratio on the void characterization}

Let us now consider the effect of the capillary ratio on the characterization of the voids (size, shape and location) formed in the first and second weft, and the differences among these voids when they are predicted by the two formulations considered here, S-D and S-B. In our analysis three different capillary ratios are considered: $C_{c a p}=1 \times 10^{-2}, C_{c a p}=$ $1 \times 10^{-1}$ and $C_{\text {cap }}=5 \times 10^{-1}$; the solutions and corresponding analysis for the former capillary ratio, $C_{c a p}=1 \times 10^{-2}$, were already discussed in the previous sub-section (Fig. 6). As it can be noticed from (21) to (30), the maximum capillary pressure in the present case is always achieved at the symmetric boundary of the warp, and it is given by: 
Stokes-Brinkman, $\beta=1.24$
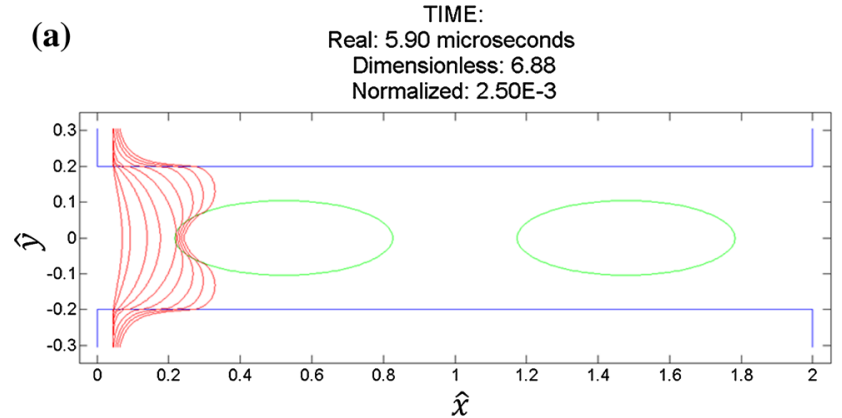

(c)

TIME:

Real: 1.37E+2 microseconds

Dimensionless: 159.16

Normalized: $5.78 \mathrm{E}-2$

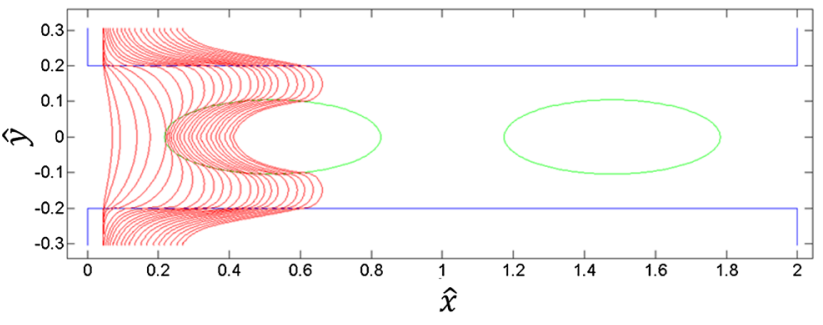

(e)

TIME:
Real: $6.80 \mathrm{E}+2$ microseconds

Dimensionless: 792.30

Normalized: $2.88 \mathrm{E}-1$



(g)

TIME:

Real: $1.29 \mathrm{E}+3$ microseconds

Dimensionless: 1504.58

Normalized: $5.47 \mathrm{E}-1$



(i)

TIME:

Real: $2.36 \mathrm{E}+3$ microseconds Dimensionless: 2752.16

Normalized: 1.0

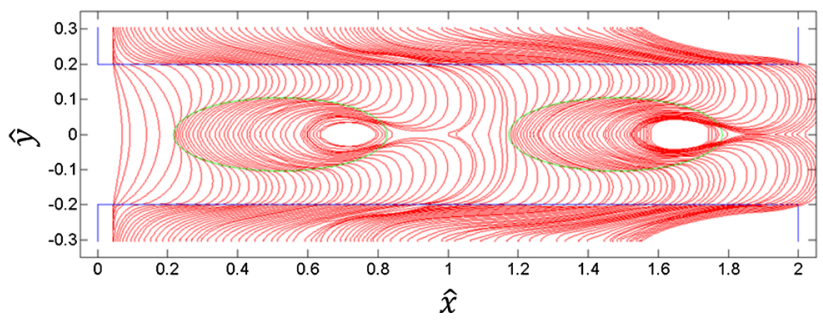

Stokes-Darcy, $\gamma=1.27$

(b)

TIME:

Real: 6.16 microseconds

Dimensionless: 7.86

Normalized: $3.03 \mathrm{E}-3$

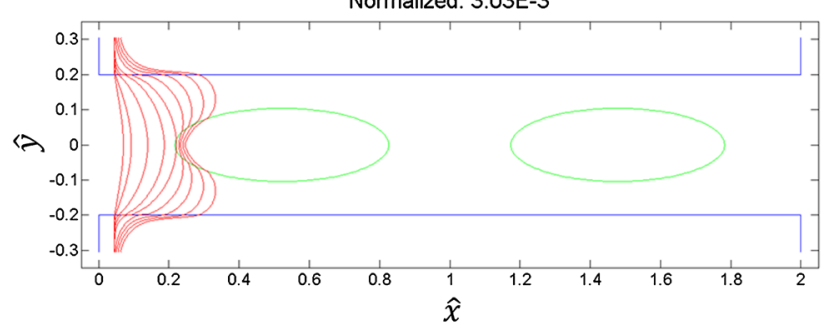

(d)

TIME:

Real: $1.34 \mathrm{E}+2$ microseconds

Dimensionless: 170.41

Normalized: $6.57 \mathrm{E}-2$

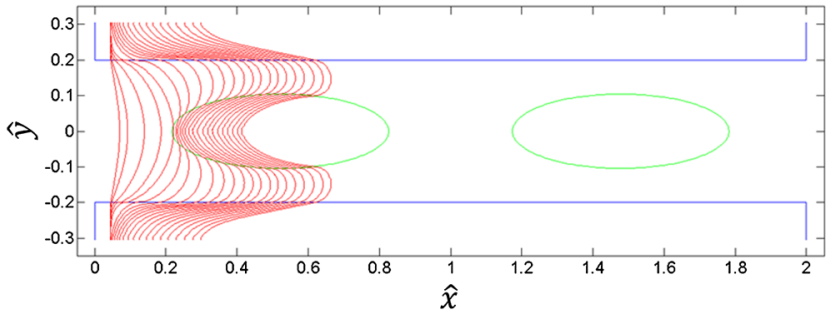

(f)

TIME:

Real: $6.26 \mathrm{E}+2$ microseconds

Dimensionless: 798.06

Normalized: 3.08E-1

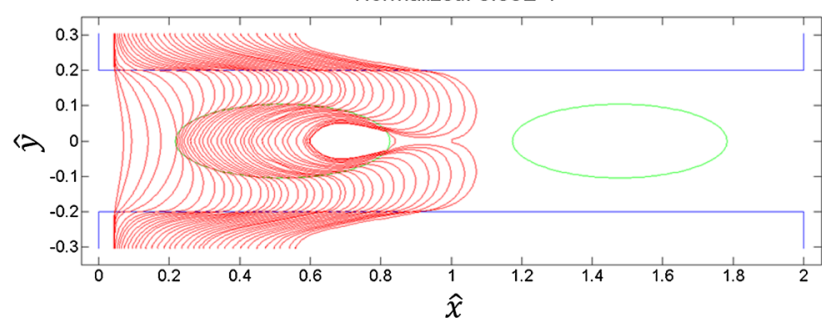

(h)

TIME:

Real: $1.16 \mathrm{E}+3$ microseconds

Dimensionless: 1482.45

Normalized: $5.72 \mathrm{E}-1$

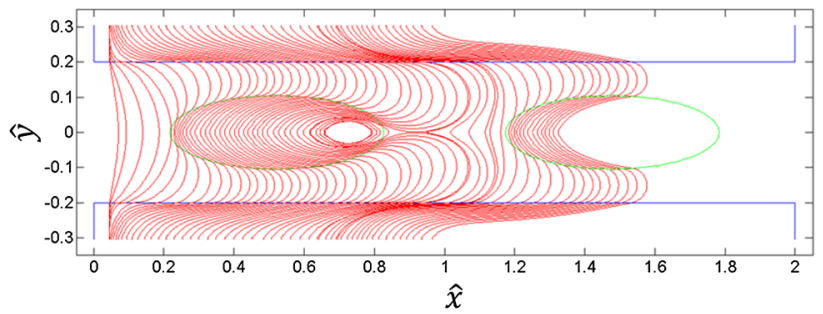

(j)

TIME:

Real: $2.03 \mathrm{E}+3$ microseconds

Dimensionless: 2593.37

Normalized: 1.0

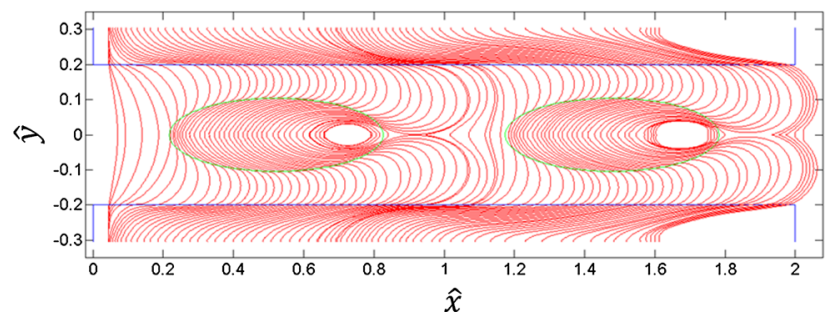

Fig. 6 Comparison of void formation between Stokes-Brinkman with $\beta=1.24$ and Stokes-Darcy with $\gamma=1.27$ 
Table 4 Characterization of final voids for S-B with $\beta=1.24$ and S-D with $\gamma=1.27$

\begin{tabular}{|c|c|c|c|c|c|c|c|}
\hline \multirow[t]{2}{*}{ Approach } & \multirow[t]{2}{*}{ Capillary ratio } & \multicolumn{3}{|c|}{ Void of first weft } & \multicolumn{3}{|c|}{ Void of second weft } \\
\hline & & Size* & Shape & Location & Size* & Shape & Location \\
\hline \multirow[t]{3}{*}{ Stokes-Brinkman } & $1.00 \times 10^{-2}$ & $7.58 \times 10^{-3}$ & 2.34 & $9.48 \times 10^{-1}$ & $9.87 \times 10^{-3}$ & 1.93 & $9.35 \times 10^{-1}$ \\
\hline & $1.00 \times 10^{-1}$ & $1.08 \times 10^{-2}$ & 2.84 & $9.83 \times 10^{-1}$ & $6.55 \times 10^{-3}$ & 3.32 & $9.87 \times 10^{-1}$ \\
\hline & $5.00 \times 10^{-1}$ & $1.97 \times 10^{-3}$ & 4.24 & $9.65 \times 10^{-1}$ & 0.00 & NA & NA \\
\hline \multirow[t]{3}{*}{ Stokes-Darcy } & $1.00 \times 10^{-2}$ & $5.08 \times 10^{-3}$ & 2.10 & $9.32 \times 10^{-1}$ & $8.01 \times 10^{-3}$ & 1.86 & $9.46 \times 10^{-1}$ \\
\hline & $1.00 \times 10^{-1}$ & $1.07 \times 10^{-2}$ & 3.10 & $9.84 \times 10^{-1}$ & $7.34 \times 10^{-3}$ & 3.59 & $9.91 \times 10^{-1}$ \\
\hline & $5.00 \times 10^{-1}$ & $4.94 \times 10^{-3}$ & 4.17 & $9.78 \times 10^{-1}$ & $2.45 \times 10^{-3}$ & 5.21 & $9.66 \times 10^{-1}$ \\
\hline
\end{tabular}

$P_{\text {cap }, \text { max }}=2 \sigma \cos (\theta)\left(1-\varepsilon_{t}\right) /\left(R \varepsilon_{t}\right)$

Consequently, in all the cases considered here, where the values of $\sigma, \theta, R$ and $\varepsilon_{t}$ are kept constants, the value of the maximum capillary pressure, $P_{\text {cap, max }}$, is always the same and therefore different values of the capillary ratio, $C_{c a p}$, corresponds to different values of the inlet pressure given by: $P_{\text {in }}=P_{\text {cap } \text { max }} / C_{\text {cap }}$.

As it was observed before in Fig. 6i (S-B) and $\mathrm{j}(\mathrm{S}-\mathrm{D})$, for $C_{\text {cap }}=1 \times 10^{-2}$ the bubble of the first weft is smaller than the one of the second weft. In such case, this happens because the first bubble supports a higher pressure considering that it is closer to the inlet and consequently experiences a higher compression. The values of the compressibility ratios obtained with both formulations, S-D and S-B, are: for the bubble of the first weft, $\psi=0.512(\mathrm{~S}-\mathrm{B})$ and $\psi=0.587$ (S-D), whereas, for the second one, $\psi=0.304(\mathrm{~S}-\mathrm{B})$ and $\psi=0.332(\mathrm{~S}-\mathrm{D})$. Figure $7 \mathrm{a}-\mathrm{d}$ show the results for the other two values of the capillary ratio $\left(C_{c a p}=1 \times 10^{-1}\right.$ and $C_{\text {cap }}=5 \times 10^{-1}$ ), where the flow in the channel has advanced enough to allow the formation of the bubbles in both wefts. For these two cases of $C_{c a p}$, the bubble at the first weft is larger than the one at the second weft for both formulations, $\mathrm{S}-\mathrm{B}$ and $\mathrm{S}-\mathrm{D}$, which is opposite to the case described before for $C_{\text {cap }}=1 \times 10^{-2}$, where the second bubble was larger than the first one. This apparent unexpected behavior is due to the larger values of the capillary ratio considered in these last two cases, $C_{c a p}=1 \times 10^{-1}$ and $C_{c a p}=5 \times 10^{-1}$, in comparison with $C_{c a p}=1 \times 10^{-2}$. From the previous analysis of the maximum capillary pressure it follows that when $P_{\text {cap, max }}$ is constant, as in the present cases, larger values of the capillary ratio, $C_{c a p}$, correspond to lower values of the inlet pressure, $P_{i n}$. At lower inlet pressure, the fluid fronts at the channel move more slowly; besides, the capillary forces always promote the weft impregnation. These two simultaneous effects result in smaller differences between the location of the fluid fronts at the channel and the location of the fluid front at the wefts before the channel fluid fronts enclose the wefts, and, consequently, in smaller initial bubbles, as $C_{c a p}$ is higher. This consequence is in turn more pronounced at the second weft due to the slower channel flow velocities obtained there regarding the ones obtained in the first weft, given the increase of the flow resistance as the fluid front progresses; accordingly, the initial void size at the second weft will be smaller than the one at the first weft. On the other hand, at a lower inlet pressure, the bubbles are subjected to lower compression, and consequently, at larger values of the capillary ratio $\left(C_{c a p}=1 \times 10^{-1}\right.$ and $\left.C_{c a p}=5 \times 10^{-1}\right)$, the initial void size is very similar to the final void size for both wefts; as the initial void is smaller for the second weft, the final void is too, as can be observed in Fig. 7 for both approaches, S-B and S-D.

The results of the void characterization obtained with both formulations, S-D and S-B, are summarized in Table 4, for all values of $C_{c a p}$ considered. As can be observed from that table, the largest voids in the first weft are obtained for $C_{\text {cap }}=1 \times 10^{-1}$ in both formulations and the smallest ones, for $C_{\text {cap }}=5 \times 10^{-1}$, with the void size corresponding to $C_{\text {cap }}=1 \times 10^{-2}$ in between them. On the other hand, for the second weft, the void is always smaller as $C_{c a p}$ is higher, with no void formation, Size $e^{*}=0$, for $C_{\text {cap }}=5 \times 10^{-1}$ when using the $S-B$ approach (see Fig. 7c), while S-D predicts the formation of a very small bubble (see Fig. 7d). Regarding the void shape, the increase of $C_{c a p}$ generates bubbles with a higher aspect ratio for both wefts using both approaches, S-D and S-B.

\subsubsection{Comparison of saturation curves}

The time evolution of the saturation curves predicted by the S-D and S-B approaches for the three capillary ratios are compared in Fig. 8a, where it can be observed a similar general behavior for all curves. Figure $8 \mathrm{~b}$ shows the saturation curve obtained with the $\mathrm{S}-\mathrm{D}$ simulation for $C_{c a p}=1 \times 10^{-1}$ in order to describe the general behavior of all cases reported in Fig. 8a. In Fig. 8b, different filling instants are highlighted using marker points, which are numerated from 0 to 5 . As can be observed, the highest saturation rate, i.e., the slope of the curve, is reached at the beginning of the injection (from 0 to 1$)$. As soon as the fluid front arrives at the first weft, 


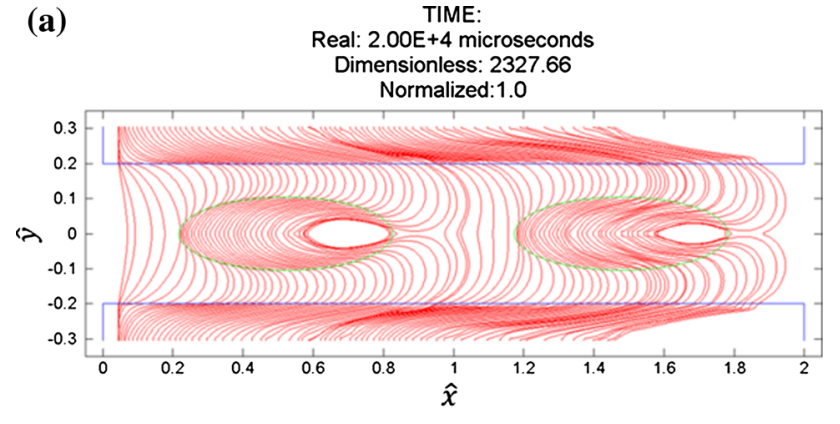

TIME:
Real:1.85E+4 microseconds
Dimensionless: 2341.41 Dimensionless: 2341.41
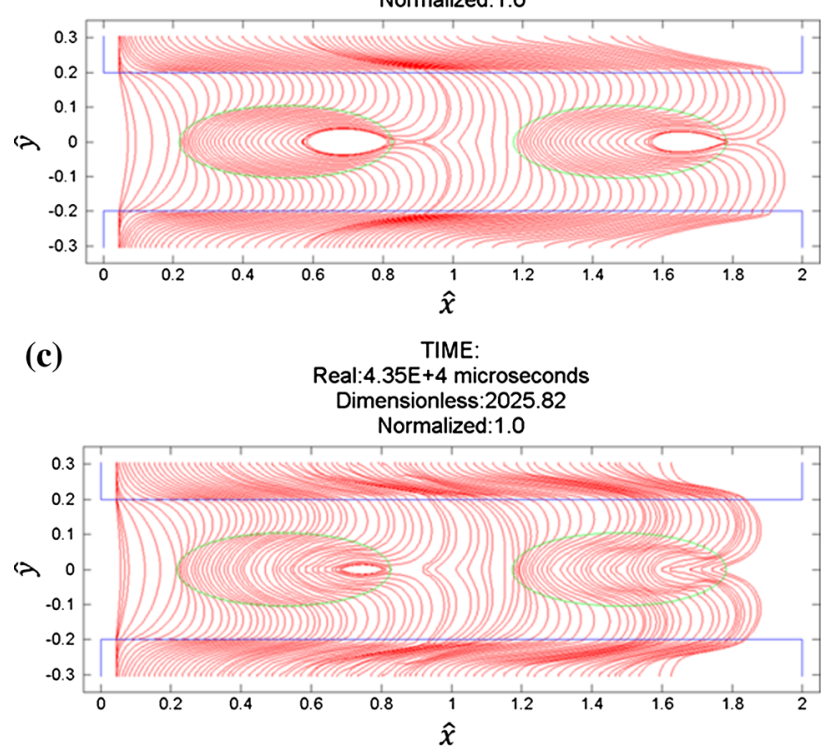

(d)



Fig. 7 Total fillings of RUC's. a $C_{c a p}=1 \times 10^{-1}$ (Stokes-Brinkman, $\beta=1.24), \mathbf{b} C_{c a p}=1 \times 10^{-1}$ (Stokes-Darcy, $\left.\gamma=1.27\right)$, c $C_{\text {cap }}=$ $5 \times 10^{-1}$ (Stokes-Brinkman, $\beta=1.24$ ), d $C_{\text {cap }}=5 \times 10^{-1}$ (StokesDarcy, $\gamma=1.27$ )

point (1), the saturation rate decreases until the merging of the channel fluid fronts (from 1 to 2 ) due to the flow resistance exerted by the first weft. When the channel fluid fronts have surrounded the first weft and encountered one another, point (2), an increase of the saturation rate can be noticed. This rate is kept almost constant until the arrival of the fluid front to the second weft (from 2 to 3 ), moment from which the saturation rate progressively decreases again until the second


Fig. 8 Saturation curves. a Comparisson Stokes-Brinkman with $\beta=$ 1.24 versus Stokes-Darcy with $\gamma=1.27$. b Stokes-Darcy for $C_{c a p}=$ $1 \times 10^{-1}$

merging of the fluid fronts (from 3 to 4 ) due to the resistance exerted by the second weft. In point (4), saturation rate barely increases, and from (4) to the end of simulation (5), saturation rate is essentially constant. The nearly constant saturation rate between 2 and 3 is expected since no porous obstacles are present in the channel between these points, in contrast with what happens between the points 1 to 2 and 3 to 4 , where the saturation rate decreases due to the presence of the first and second weft, respectively.

According to Fig. 8a, for $C_{c a p}=1 \times 10^{-2}$ and $C_{c a p}=$ $1 \times 10^{-1}$, the saturation curves predicted by both approaches (S-D and S-B) are almost identical, but the final saturation is slightly higher for the S-D result (see detail in Fig. 8a). The highest differences between the saturation curves of both approaches (S-D and S-B) are observed for $C_{c a p}=5 \times 10^{-1}$, where the capillary effects are the most relevant; in this case, the final RUC saturation is barely higher for the S-B scheme (see detail in Fig. 8a).

\subsection{Influence of matching conditions on the void formation for the Stokes-Brinkman approach}

\subsubsection{Influence of the jump stress coefficient}

Let us first consider the influence of the jump stress coefficient, $\beta$, on the void formation by comparing the results obtained from Series $1(\beta=1.24)$ and $2(\beta=0.70)$, for the 

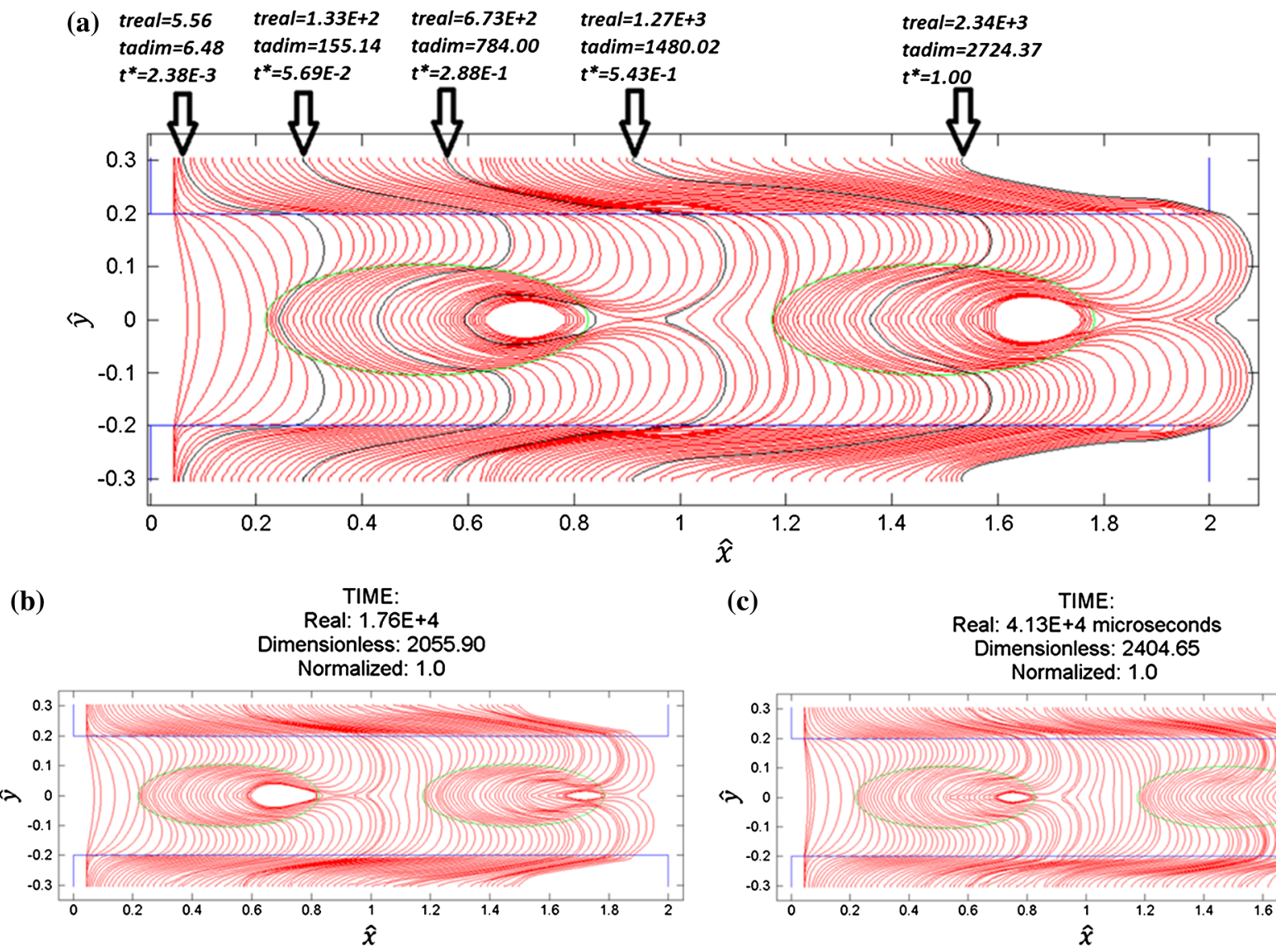

(c)

TIME:

Real $4.13 E+4$ microseconds Dimensionless: 2404.65

Normalized: 1.0



Fig. 9 Total fillings of RUC's for Stokes-Brinkman with $\beta=0.7$. a $C_{c a p}=1 \times 10^{-2}$, b $C_{\text {cap }}=1 \times 10^{-1}$, c $C_{\text {cap }}=5 \times 10^{-1}$

Table 5 Influence of $\beta$ and $C_{c a p}$ on the void size

\begin{tabular}{|c|c|c|c|c|c|c|}
\hline \multirow[t]{3}{*}{ Capillary ratio } & \multicolumn{6}{|l|}{ Void size } \\
\hline & \multicolumn{2}{|l|}{ First weft } & \multicolumn{2}{|l|}{ Second weft } & \multicolumn{2}{|l|}{ Total } \\
\hline & $\beta=1.24$ & $\beta=0.70$ & $\beta=1.24$ & $\beta=0.70$ & $\beta=1.24$ & $\beta=0.70$ \\
\hline $1.00 \times 10^{-2}$ & $7.582 \times 10^{-3}$ & $6.573 \times 10^{-3}$ & $9.866 \times 10^{-3}$ & $8.796 \times 10^{-3}$ & $1.745 \times 10^{-2}$ & $1.537 \times 10^{-2}$ \\
\hline $1.00 \times 10^{-1}$ & $1.077 \times 10^{-2}$ & $1.039 \times 10^{-2}$ & $6.548 \times 10^{-3}$ & $2.150 \times 10^{-3}$ & $1.732 \times 10^{-2}$ & $1.254 \times 10^{-2}$ \\
\hline $5.00 \times 10^{-1}$ & $1.973 \times 10^{-3}$ & $1.927 \times 10^{-3}$ & 0.00 & 0.00 & $1.973 \times 10^{-3}$ & $1.927 \times 10^{-3}$ \\
\hline
\end{tabular}

three different values of $C_{c a p}$ considered here. The total fillings of the RUC for Serie 1 and the different values of $C_{\text {cap }}$ were already analyzed in the previous section and reported in Fig. 6i $\left(C_{c a p}=1 \times 10^{-2}\right)$, $7 \mathrm{a}\left(C_{c a p}=1 \times 10^{-1}\right)$, and $7 \mathrm{c}\left(C_{\text {cap }}=5 \times 10^{-1}\right)$. Equivalent results for Serie 2 are shown in Fig. 9a-c, where, in the case of $C_{c a p}=1 \times 10^{-2}$ (Fig. 9a), different fluid fronts with positions in the channel corresponding to the same ones reported in the first column of Fig. 6 for the Serie 1 are highlighted using arrows. As can be seen from these figures (first column of Figs. 6 and 9a), the real, dimensionless and normalized arrival times corresponding to these positions are shorter for $\beta=0.70$ (Fig. 9a).

In Table 5, the effect of $C_{c a p}$ on the void size at the first and second wefts for both values of $\beta$ ( $\beta=1.24$ and $\beta=0.70)$ is presented, with the smaller voids found for $\beta=0.70$ in both wefts; the void size difference between both values of $\beta$ is less significant for $C_{c a p}=5 \times 10^{-1}$, i.e. the larger capillary ratio examined here. Although the size of the first bubble is not a monotonic function of $C_{c a p}$ for both values of $\beta$, with the smaller voids corresponding to $C_{c a p}=5 \times 10^{-1}$ and the larger ones, to $C_{c a p}=1 \times 10^{-1}$, the size of the second bubble is a decreasing monotonic function of $C_{c a p}$, and the resulting total void size, which is the sum of the size of both bubbles, also decreases with $C_{c a p}$. This last behavior is in agreement with results previously reported in the literature [30], where unidirectional macroscopic simulations were carried out using the software LIMS and it was found that the saturated length corresponding to the arrival of the 
Fig. 10 Total filling of the RUC for the continuous-stress condition and $C_{\text {cap }}=1 \times 10^{-2}$

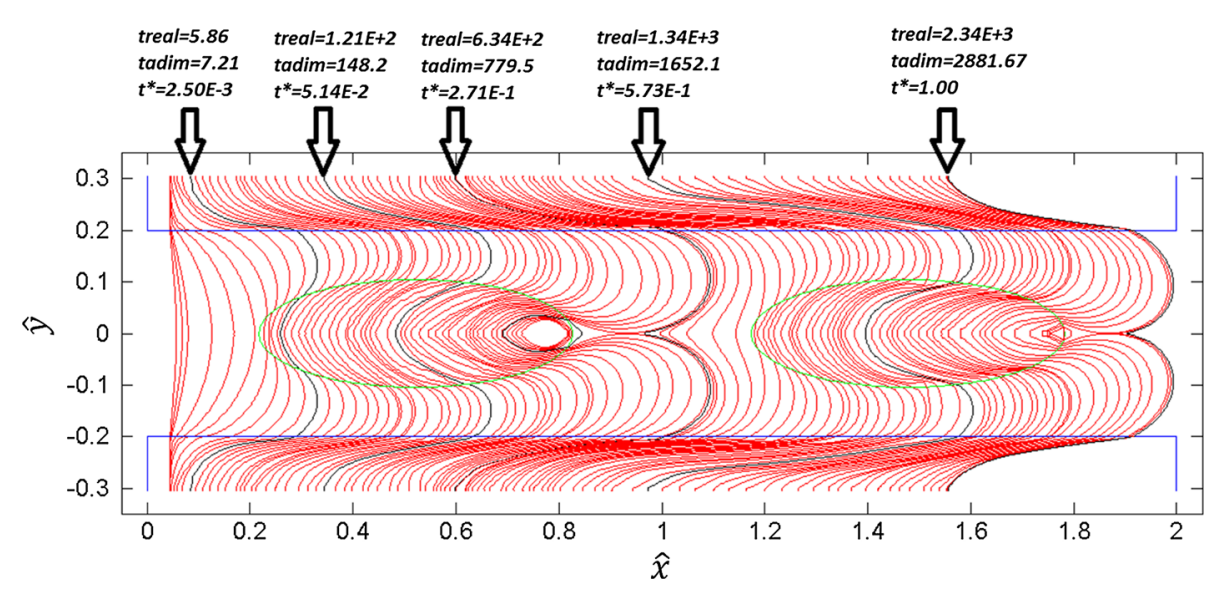

Table 6 Influence of $\beta$ and $C_{\text {cap }}$ on the void shape

\begin{tabular}{|c|c|c|c|c|}
\hline \multirow[t]{3}{*}{ Capillary ratio } & \multicolumn{4}{|c|}{ Void aspect ratio } \\
\hline & \multicolumn{2}{|l|}{ First weft } & \multicolumn{2}{|c|}{ Second weft } \\
\hline & $\beta=1.24$ & $\beta=0.70$ & $\beta=1.24$ & $\beta=0.70$ \\
\hline $1.00 \times 10^{-2}$ & 2.34 & 2.22 & 1.93 & 1.90 \\
\hline $1.00 \times 10^{-1}$ & 2.84 & 3.19 & 3.32 & 4.18 \\
\hline $5.00 \times 10^{-1}$ & 4.24 & 3.48 & NA & NA \\
\hline
\end{tabular}

fluid front to the end of a cavity is larger as $C_{c a p}$ increases, which, from a mesoscopic viewpoint, can be interpreted as the reduction of the total void size with the increment of $C_{c a p}$ for a given position along the cavity.

In general, the void shapes obtained in this work are physically consistent with some experimental works. For instance, Hamidi et al. $[20,21]$ studied the void morphology in the Resin Transfer Molding process (RTM), observing different void shapes inside the bundles:

- Cigar-shaped bubbles with a very high aspect ratio, as those ones reported in Figs. 7c-first weft, 7d-second weft, and $9 \mathrm{~b}$-second weft.

- Elliptical bubbles with a lower aspect ratio than the previous ones, see for example Figs. 6i, 7a, b, 9a, b-first weft.

- Almost circular bubbles, which are less frequent, having an aspect ratio close to one, see Fig. 10

According to the results in Table 6, the void aspect ratio at the first and second wefts increases with $C_{c a p}$ for both jump stress coefficients, $\beta=0.70$ and $\beta=1.24$. In the case of $C_{\text {cap }}=1 \times 10^{-2}$, the void aspect ratio does not change considerably with $\beta$ for both wefts, while for $C_{c a p}=$ $1 \times 10^{-1}$, a significant reduction of the aspect ratio with the increase of $\beta$ is found. On the other hand, when $C_{c a p}=$ $5 \times 10^{-1}$, only the first bubble is formed for both values of $\beta$ and the aspect ratio increases with $\beta$.
The obtained numerical results do not reveal a relevant influence of $\beta$ and $C_{c a p}$ on the location of the void, which is always formed at the rear edge of the weft. This is also in agreement with the experimental results of Hamidi et al. [21], where radial injections in a circular mold were conducted, founding that the most of voids were formed at the rear edge of the tows with respect to the flow direction, both inside the tow and in the transition tow-channel. Other numerical works, [24, 25,43,54], have also predicted the void formation at similar locations.

In summary, it can be concluded that the process of void formation by mechanical entrapment of air is defined by a dynamic balance between the inlet and capillary pressures, which is characterized by the capillary ratio, $C_{c a p}$. The void size and shape are also influenced by the magnitude of $C_{c a p}$, as well as by the value of the jump stress coefficient, $\beta$. On the other hand, the void location appears to be independent on both $C_{c a p}$ and $\beta$, since the voids are always located at the rear edge of the wefts, which is in agreement with previous numerical and experimental works.

\subsubsection{Comparison of void size between continuous stress and jump stress simulations}

The influence of the type of interface condition on the void formation for $C_{c a p}=1 \times 10^{-2}$ is studied by comparing results of Serie 2 (stress jump condition, $\beta=0.7$ ) and Serie 3 (continuous stress condition, $\beta=0$ ). The total RUC filling for the continuous stress condition is presented in Fig. 10, where the fluid fronts having the same positions in the channel to those ones depicted in Fig. 9a are highlighted by the corresponding arrows.

The comparison between both simulations (Fig. 9a vs. Fig. 10) indicates that for the same fluid front position in the channel, the positions of the fluid fronts in the warps and wefts are more advanced in the continuous-stress case ( $\beta=0)$, and consequently, the initial size of the first and second bubbles, corresponding to the instant when the chan- 
nel fluid fronts completely enclose the first and second weft, respectively, is smaller in the continuous stress conditions. The initial and final void size at the first weft in the case of the jump-stress condition, Fig. 9a, is Size $e^{*}=1.52 \times 10^{-2}$ and Size $e^{*}=6.57 \times 10^{-3}$, respectively; on the other hand, these values for the continuous-stress condition, Fig. 10, are Size $e^{*}=6.30 \times 10^{-3}$ and Size $e^{*}=2.69 \times 10^{-3}$. In the case of the jump-stress condition the compressibility ratio is $\psi=0.567$, whereas for the continuous stress this value is $\psi=0.573$. Accordingly, the void compression does not have a relevant influence in the difference between the final void sizes for these two cases (continuous-stress and jumpstress) since the compressibility ratios are almost the same, and the main reason of this difference is the initial void size, which is smaller in the case of $\beta=0$, leading to a smaller final void in such a case.

A similar behavior can be appreciated for the void size at the second weft, namely, the initial void is smaller in the continuous-stress case and the compressibility ratio is almost the same in both cases (continuous-stress and jump-stress); the difference between the final void sizes at the second weft for these two cases is even higher than at the first weft, with $S i z e^{*}=8.80 \times 10^{-3}$ in the case of the jump-stress condition, and $S_{i z} e^{*}=2.02 \times 10^{-4}$ for the continuous-stress condition.

\subsubsection{Comparison of saturation curves between continuous stress and jump stress simulations}

Finally, let us analyze the differences between the predicted saturation curves of both conditions, continuous stress and jump stress. Saturation curves are very important on the quantitative description of the time and space evolution of the saturated volume, and they can be very useful for the comparison of filling processes at different filling instants. The obtained saturation curves for the continuous (C-S) and jump (J-S) stress conditions are compared in Fig. 11. From this figure, it can be observed that the time evolution of the corresponding saturation curves is very similar to the one presented in Fig. 8b, with the C-S condition predicting a higher value of saturation at any normalized time, $t^{*}=t / t_{\text {filling }}$.

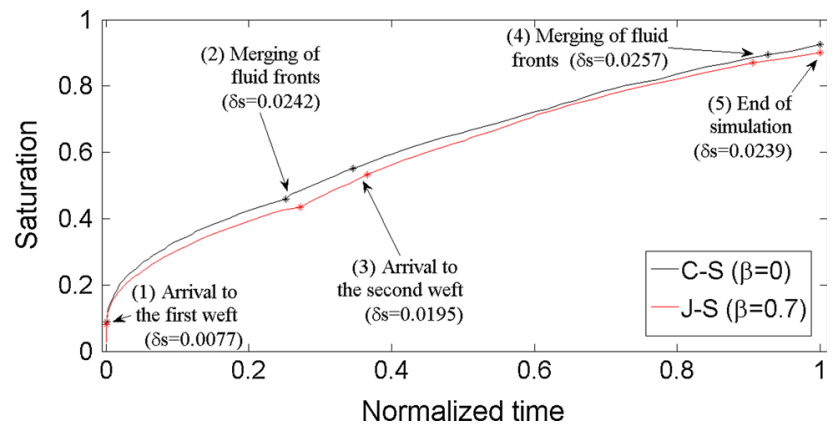

Fig. 11 Comparison of saturation curves for continuous and jump stress conditions and $C_{\text {cap }}=1 \times 10^{-2}$
In the saturation curves shown in Fig. 11, the representative filling instants reported in Fig. $8 \mathrm{~b}(i=1,2,3,4,5)$ are highlighted by the arrows. The saturation difference between the both curves ( $\mathrm{C}-\mathrm{S}$ and $\mathrm{J}-\mathrm{S}$ conditions), defined as $d s=s_{i}^{\text {cont }}-s_{i}^{j u m p}$, is not the same for all filling instants . Here, $s_{i}^{\text {cont }}$ and $s_{i}^{j \text { ump }}$ stand for the RUC saturation obtained with the $\mathrm{C}-\mathrm{S}$ and $\mathrm{J}-\mathrm{S}$ simulations, respectively. It is very important to take into account that, in this case, $d s$ is not referred to the saturation difference for a determined value of the normalized time, $t^{*}$, but to the saturation difference corresponding to any of the instants highlighted in Fig. 11, which do not necessarily correspond to the same values of $t^{*}$ in both simulations, C-S and J-S.

In the first filling instants (1), when the flow reaches the first weft, the RUC saturation is very similar for both simulations ( $d s$ is very small) because it is primarily determined by the saturation of the channel, which is almost the same in both cases. During the impregnation of the first weft (from 1 to 2), $d s$ increases until the merging of the channel fluid fronts at (2), due to the higher weft saturation obtained in the $\mathrm{C}-\mathrm{S}$ simulation during this period, as commented before. The change of the saturated volume of the first weft is not completely defined when the channel fluid fronts enclose it since the void undergoes a continuous compression during the filling process of the RUC until the partial equilibrium is reached. Therefore, the change of the saturated volume of the first weft from the time of merging of the fluid fronts to the time of arrival of the fluid front to the second weft (from 2 to 3 ) is due to the compression of the bubble. Given that during this period the filling of the channel and the warps is almost identical for the C-S and J-S simulations, the change of $d s$ is essentially determined by the compression of the bubble. Accordingly, as the compressibility ratio, $\psi$, of C-S and J-S is very similar for the first bubble, as mentioned before, and considering the definition of $\psi$ in (63), the change of the volume of the bubble is larger in the case of the larger initial void size, which corresponds to the J-S simulation. Therefore, the change of the saturated volume of the first weft is also larger for the J-S simulation between the time instants analyzed (from 2 to 3 ) and this leads to the reduction of the saturation difference, $d s$ (see Fig. 11).

In the C-S simulation, a larger infiltration at the second weft occurs between the instant of the arrival of the fluid front to this weft and the instant of merging of the channel fluid fronts to form the second bubble (from 3 to 4). During this time interval, both simulations $(\mathrm{C}-\mathrm{S}$ and $\mathrm{J}-\mathrm{S})$ predict almost the same changes on the saturated volume in the channels and the warps, and the change on the saturated volume in the first weft due to the void compression is negligible in comparison with the change of the saturated volume in the second weft. These factors lead to an increment of $d s$ that is mainly determined by the change of the saturation in the second weft, 
which, in turn, is larger for the C-S condition as mentioned before (see Fig. 11). At the end of the simulation, the RUC saturation is $s=0.928$ for $\mathrm{C}-\mathrm{S}$ and $s=0.904$ for J-S.

\section{Conclusions}

In this work, BEM techniques have been implemented for the simulation of voids formation in dual-scale fibrous reinforcements using Stokes-Darcy (S-D) and Stokes-Brinkman (S-B) mathematical formulations. The BEM solution for the S-D formulation was previously validated in [43], while a coupled Stokes-Brinkman problem with analytical solution was used here to validate the developed BEM/DR-BEM scheme (BEM for the Stokes domain and DR-BEM for the Brinkman domain), obtaining a very good accuracy. The error of the numerical solution increases with the magnitude of the inverse Darcian number, $\chi^{2}$, for all values of the jump stress coefficient, $\beta$, considered here, and decreases with the magnitude of $\beta$ for each value of $\chi^{2}$, except for the smallest one, $\chi^{2}=18$, where the error increases with $\beta$ (see Table 2 ).

The present numerical solution predicts a reduction of the Stokes velocity in the channel and of the boundary layer thickness in the porous medium with the increase of $\beta$ and $\chi^{2}$, which is in agreement with the results in [58]. Moreover, our solution is also compared with a FEM solution previously reported in [23], finding that both approaches converge satisfactorily to the analytical solution, with larger errors in the Brinkman domain than in the Stokes domain in the present BEM/DR-BEM scheme, contrarily to the FEM scheme of [23] where the errors in the Stokes domain are greater. Conversely to the FEM solution, no numerical oscillations in the boundary layer were obtained with the present BEM/DRBEM scheme.

The problem of void formation during the filling of two adjacent RUC's, for three different capillary ratios, $C_{\text {cap }}=\left[1 \times 10^{-2}, 1 \times 10^{-1}, 5 \times 10^{-1}\right]$, was analyzed by using Stokes-Darcy (S-D) and Stokes-Brinkman (S-B) formulations, resulting in a similar behavior of the void size and shape with $C_{c a p}$ for both formulations. At the first weft, the change of void size with $C_{c a p}$ is not monotonous, with the largest voids obtained for $C_{\text {cap }}=1 \times 10^{-1}$, the smallest ones for $C_{c a p}=5 \times 10^{-1}$, and the intermediate ones for $C_{\text {cap }}=1 \times 10^{-2}$. For the second weft, the void is smaller as $C_{\text {cap }}$ increases. The total void size, which is the sum of the size of both bubbles, also decreases with $C_{c a p}$, which is in agreement with the results of [30]. On the other hand, the void aspect ratio in both wefts increases with $C_{c a p}$.

The results of the RUC's fillings also allow concluding that the differences between the S-D and S-B formulations depend on the capillary effects. For instance, in the particular case when the capillary effects are weak, i.e., $C_{\text {cap }}=1 \times$ $10^{-2}$, the S-D formulation predicts smaller voids and lower aspect ratios than $\mathrm{S}-\mathrm{B}$, in both wefts. On the other hand, for a same value of $C_{c a p}$, similar saturation curves were obtained with both formulations, S-D and S-B, with exception of the case when the capillary effects are dominant, i.e., $C_{c a p}=$ $5 \times 10^{-1}$, where important differences between the saturation curves of both formulations were appreciated.

According to the present results, the type of matching conditions considered in the Stokes-Brinkman formulation affects the void size and shape. When using the jump stress condition $(\mathrm{J}-\mathrm{S})$, the smallest voids at both wefts were obtained for the lowest value of the jump stress coefficient, $\beta=0.7$, for all values of $C_{c a p}$, with the exception of the case of the second weft when $C_{c a p}=5 \times 10^{-1}$ (when capillary effects are dominant), where no void formation was obtained for the two values of the jump stress coefficient $(\beta=1.24$ and $\beta=0.7)$. Regarding the void shape, the aspect ratio always increases with $C_{c a p}$ for each value of $\beta$ in both wefts (see Table 6). According to the BEM/DR-BEM simulations, the void location is not significantly affected by $C_{c a p}$ and $\beta$, and is formed at the rear edge of the weft.

The general behavior of the void size and aspect ratio with $C_{c a p}$ in both wefts, which was described above, did not change with $\beta$, as well as the decreasing behavior of the total void content with $C_{c a p}$, which was obtained in all cases of the S-B formulation. On the other hand, for $C_{c a p}=1 \times 10^{-2}$, the lowest void content was obtained for the continuous-stress condition (C-S), which is consistent with the larger saturation predicted by the $\mathrm{C}-\mathrm{S}$ condition for all filling instants in comparison with the one predicted by J-S. Despite the saturation values differ in all filling instants, both saturations curves, C-S and J-S, showed a similar behavior.

Acknowledgements The financial support provided by Grant 567 Colciencias (Departamento Administrativo de Ciencia, Tecnología e Innovación) and Grant 2015 Enlazamundos Sapiencia (Agencia de Educación Superior de Medellín) is gratefully acknowledged.

Open Access This article is distributed under the terms of the Creative Commons Attribution 4.0 International License (http://creativecomm ons.org/licenses/by/4.0/), which permits unrestricted use, distribution, and reproduction in any medium, provided you give appropriate credit to the original author(s) and the source, provide a link to the Creative Commons license, and indicate if changes were made.

\section{References}

1. Amico S, Lekakou C (2001) An experimental study of the permeability and capillary pressure in resin-transfer moulding. Compos Sci Technol 61(13):1945-1959. doi:10.1016/ S0266-3538(01)00104-X

2. Angot P (2011) On the well-posed coupling between free fluid and porous viscous flows. Appl Math Lett 24(6):803-810

3. Bathe KJ (2001) The inf-sup condition and its evaluation for mixed finite element methods. Comput Struct 79(2):243-252. doi:10. 1016/S0045-7949(00)00123-1

4. Breugem WP (2007) The effective viscosity of a channel-type porous medium. Phys Fluids 19(10):1-16. doi:10.1063/1.2792323 
5. Chandrupatla T, Osler T (2010) The perimeter of an ellipse. Math Sci 35:122-131

6. Clements DL (1981) Boundary value problems governed by second order elliptic systems, 1st edn. Pitman Advanced Publishing Program, California

7. Devalve C, Pitchumani R (2013) Simulation of void formation in liquid composite molding processes. Compos A 51:22-32. doi:10. 1016/j.compositesa.2013.03.016

8. Discacciati M, Miglio E, Quarteroni A (2002) Mathematical and numerical models for coupling surface and groundwater flows. Appl Numer Math 43(1-2):57-74. doi:10.1016/ S0168-9274(02)00125-3

9. Durlofsky L, Brady JF (1987) Analysis of the Brinkman equation as a model for flow in porous media. Phys Fluids 30(11):3329-3341. doi:10.1063/1.866465

10. Florez WF, Power H (2002) DRM mult idomain mass conservative interpolation approach for the BEM solution of the two-dimensional Navier-Stokes equations. Comput Math Appl 43:457-472

11. Gangloff JJ, Hwang WR, Advani SG (2014) Characterization of bubble mobility in channel flow with fibrous porous media walls. Int J Multiph Flow 60:76-86. doi:10.1016/j.ijmultiphaseflow.2013. 12.003

12. Gantois R, Cantarel A, Dusserre G (2013) BEM-based models to simulate the resin flow at macroscale and microscale in LCM processes. Polym Compos 34(8):1235-1244. doi:10.1002/pc.22531

13. Gantois R, Cantarel A, Dusserre G, Félices JN, Schmidt F (2011) Mold filling simulation of resin transfer molding combining BEM and level set method. Appl Mech Mater 62:57-65. doi:10.4028/ www.scientific.net/AMM.62.57

14. García J (2000) Gestión del frente de avance en la modelización numérica del conformado de Transferencia de Resina (RTM). Ph.D. thesis, PHD Thesis, Universidad Politécnica de Valencia

15. García JA, Gascón L, Chinesta F (2003) A fixed mesh numerical method for modelling the flow in liquid composites moulding processes using a volume of fluid technique. Comput Methods Appl Mech Eng 192(7-8):877-893. doi:10.1016/ S0045-7825(02)00604-7

16. Gebart B (1992) Permeability of unidirectional reinforcements for RTM. J Compos Mater 26(8):1100-1133

17. Givler R, Sa Altobelli (1994) A determination of the effective viscosity for the Brinkman-Forchheimer flow model. J Fluid Mech 258(1934):355-370. doi:10.4103/0250-474X.89772

18. Goldberg M, Chen C (1997) Discrete projection methods for integral equations, 1 st edn. WIT Press, Southampton

19. Gourichon B, Binetruy C, Krawczak P (2006) A new numerical procedure to predict dynamic void content in liquid composite molding. Compos A 37(11):1961-1969. doi:10.1016/j. compositesa.2005.12.017

20. Hamidi YK, Aktas L, Altan MC (2004) Formation of microscopic voids in resin transfer molded composites. J Eng Mater Technol 126(4):420-426. doi:10.1115/1.1789958

21. Hamidi YK, Aktas L, Altan MC (2005) Three-dimensional features of void morphology in resin transfer molded composites. Compos Sci Technol 65(7-8):1306-1320. doi:10.1016/j.compscitech.2005. 01.001

22. Huang LH, Chiang IL, Song CH (1997) A re-investigation of laminar channel flow passing over porous bed. J Chin Inst Eng 20(4):435-441

23. Hwang WR, Advani SG (2010) Numerical simulations of StokesBrinkman equations for permeability prediction of dual scale fibrous porous media. Phys Fluids 22(11):1-14. doi:10.1063/1. 3484273

24. Jinlian H, Yi L, Xueming S (2004) Study on void formation in multi-layer woven fabrics. Compos A 35(5):595-603. doi:10.1016/ j.compositesa.2003.11.007
25. Kang MK, Lee WI, Hahn HT (2000) Formation of microvoids during resin-transfer molding process. Compos Sci Technol 60:24272434

26. Kohr M, Sekhar GPR, Blake JR (2007) Green's function of the Brinkman equation in a 2D anisotropic case. IMA J Appl Math 73(2):374-392. doi:10.1093/imamat/hxm023

27. Kohr M, Wendland WL (2009) Sekhar GPR (2009) Boundary integral equations for two-dimensional low Reynolds number flow past a porous body. Math Methods Appl Sci 32:922-962. doi:10.1002/ mma.1074

28. Krotkiewski M, Ligaarden IS, Lie KA, Schmid DW (2011) On the Importance of the Stokes-Brinkman equations for computing effective permeability in karst reservoirs. Commun Comput Phys 10(5):1315-1332. doi:10.4208/cicp.290610.020211a

29. Larson R, Higdon J (1989) A periodic grain consolidation model of porous media. Phys Fluids A 1:38-46

30. Lawrence JM, Neacsu V, Advani SG (2009) Modeling the impact of capillary pressure and air entrapment on fiber tow saturation during resin infusion in LCM. Compos A 40(8):1053-1064. doi:10.1016/ j.compositesa.2009.04.013

31. Layton W, Schieweck F, Yotov I (2002) Coupling fluid flow with porous media flow. SIAM J Numer Anal 40(6):2195-2218. doi:10. 1137/S0036142901392766

32. Lebel F (2012) Controle de la Fabrication des Composites par injection sur renforts. Ph.D. thesis, Université de Montréal

33. Lundström T, Stenberg R, Bergström R, Partanen H, Birkeland P (2000) In-plane permeability measurements: a nordic round-robin study. Compos A 31(1):29-43. doi:10.1016/ S1359-835X(99)00058-5

34. Martys N, Bentz DP, Garboczi EJ (1994) Computer simulation study of the effective viscosity in Brinkman's equation. Phys Fluids 6(4):1434-1439. doi:10.1063/1.868258

35. Masoodi R, Pillai KM (2012) A general formula for capillary suction-pressure in porous media. J Porous Media 15(8):775-783. doi:10.1615/JPorMedia.v15.i8.60

36. Mu M, Zhu X (2009) Decoupled schemes for a non-stationary mixed Stokes-Darcy model. Math Comput 79(270):707-731. doi:10.1090/S0025-5718-09-02302-3

37. Mu MO, Xu J (2007) A two-grid method of a mixed Stokes-Darcy model for coupling fluid flow with porous media flow. SIAM J Numer Anal 45(5):1801-1813

38. Neacsu V, Abu Obaid A, Advani SG (2006) Spontaneous radial capillary impregnation across a bank of aligned micro-cylinders. Part I: theory and model development. Int J Multiphase Flow 32(6):661676. doi:10.1016/j.ijmultiphaseflow.2006.02.006

39. Neale G, Nader W (1974) Practical significance of Brinkman's extension of Darcy's law: coupled parallel flows within a channel and a bounding porous medium. Can J Chem Eng 52:475-478

40. Ochoa-tapia J, Whitaker S (1995) Momentum transfer at the boundary between a porous medium and a homogeneous fluid I. Theoretical development. Int. J Heat Mass Transf 38(14):26352646. doi:10.1016/S0017-9310(96)00250-5

41. Ochoa-tapia JA, Whitaker S (1995) Momentum transfer at the boundary between a porous medium and a homogeneous fluid. II. Comparison with experiment. Int. J Heat Mass Transf 38(14):26472655. doi:10.1016/0017-9310(94)00347-X

42. Partridge PPW, Brebbia CAC, Wrobel LLC (1992) The dual reciprocity boundary element method. Computational Mechanics Publication, London, England

43. Patiño Arcila ID, Power H, Nieto Londoño C, Flórez Escobar WF (2016) Boundary element simulation of void formation in fibrous reinforcements based on the Stokes-Darcy formulation. Comput Methods Appl Mech Eng 304:265-293. doi:10.1016/j.cma.2016. 02.010 
44. Posrikidis C (2002) A practical guide to boundary element methods, 1st edn. Chapman \& Hall/ CRC, London

45. Power H, Wrobel L (1995) Boundary integral methods in fluid mechanics, 1st edn. Computational Mechanics Publications, Southhampton

46. Prakash J, Raja Sekhar GP (2011) Arbitrary oscillatory Stokes flow past a porous sphere using Brinkman model. Meccanica 47(5):1079-1095. doi:10.1007/s11012-011-9494-1

47. Prakash J, Raja Sekhar GP, Kohr M (2011) Stokes flow of an assemblage of porous particles: stress jump condition. Z Angew Math Phys 62(6):1027-1046. doi:10.1007/s00033-011-0123-6

48. Richardson J, Power H (1996) A BEM analysis of creeping flow past two porous bodies of arbitrary shape. Eng Anal Bound Elem 17:193-204

49. Schell J, Deleglise M, Binetruy C, Krawczak P, Ermanni P (2007) Numerical prediction and experimental characterisation of mesoscale-voids in liquid composite moulding. Compos A 38(12):24602470. doi:10.1016/j.compositesa.2007.08.005

50. Shou D, Ye L, Tang Y, Fan J, Ding F (2013) Transverse permeability determination of dual-scale fibrous materials. Int J Heat Mass Transf 58:532-539. doi:10.1016/j.ijheatmasstransfer.2012.11.017

51. Shou D, Ye L, Tang Y, Fan J, Ding F (2015) Longitudinal permeability determination of dual-scale fibrous materials. Compos A 68:42-46. doi:10.1016/j.ijheatmasstransfer.2012.11.017

52. Shuaib N, Power H, Hibberd S (2009) BEM solution of thin film flows on an inclined plane with a bottom outlet. Eng Anal Bound Elem 33(3):388-398. doi:10.1016/j.enganabound.2008.06.007

53. Soukane S, Trochu F (2006) Application of the level set method to the simulation of resin transfer molding. Compos Sci Technol 66(7-8):1067-1080. doi:10.1016/j.compscitech.2005.03.001

54. Spaid MAA, Phelan FR Jr (1998) Modeling void formation dynamics in fibrous porous media with the lattice Boltzmann method. Compos A 29A:749-755

55. Sun W, Andrade J, Rudnicki W (2011) Multiscale method for characterization of porous microstructures and their impact on macroscopic effective permeability. Int J Numer Methods Eng 88(12):1260-1279. doi:10.1002/nme.3220

56. Tamayol A, Khosla A, Gray B, Bahrami M (2012) Creeping flow through ordered arrays of micro-cylinders embedded in a rectangular minichannel. Int J Heat Mass Transf 55(15-16):3900-3908. doi:10.1016/j.ijheatmasstransfer.2012.03.008

57. Tamayol A, Yeom J, Akbari M, Bahrami M (2013) Low Reynolds number flows across ordered arrays of micro-cylinders embedded in a rectangular micro/minichannel. Int J Heat Mass Transf 58(12):420-426. doi:10.1016/j.ijheatmasstransfer.2012.10.077
58. Tan H, Pillai KM (2009) Finite element implementation of stressjump and stress-continuity conditions at porous-medium, clearfluid interface. Comput Fluids 38(6):1118-1131. doi:10.1016/j. compfluid.2008.11.006

59. Tan H, Pillai KM (2012) Multiscale modeling of unsaturated flow in dual-scale fiber preforms of liquid composite molding I: Isothermal flows. Compos A 43(1):29-44. doi:10.1016/j.compositesa.2010. 12.013

60. Tan H, Pillai KM (2012) Multiscale modeling of unsaturated flow of dual-scale fiber preform in liquid composite molding II: Non-isothermal flows. Compos A 43(1):14-28. doi:10.1016/ j.compositesa.2011.06.012

61. Telles J (1987) A self adaptative coordinate transformation for efficient numerical evaluation of general boundary element integrals. Int J Numer Methods Eng 24(5):959-973

62. Em Ului (2011) Boundary integral equations for the problem of 2 D Brinkman flow past several voids. Stud Univ Babes-Bolyai Math LV I(1):179-193

63. Valdes-Parada FJ, Alberto Ochoa-Tapia J, Alvarez-Ramirez J (2007) On the effective viscosity for the Darcy-Brinkman equation. Physica A 385(1):69-79. doi:10.1016/j.physa.2007.06.012

64. Valdés-Parada FJ, Goyeau B, Ochoa-Tapia JA (2006) Momentum stress jump condition at the fluid-porous boundary: prediction of the jump coefficient. Aiche Conf Transp Process Multiphase Syst I I(108h): $1-8$

65. Valdés-Parada FJ, Goyeau B, Ochoa-Tapia JA (2007) Jump momentum boundary condition at a fluid-porous dividing surface: derivation of the closure problem. Chem Eng Sci 62(15):40254039. doi:10.1016/j.ces.2007.04.042

66. Whitaker S (1986) Flow in porous media I: a theoretical derivation of Darcy's law. Transp Porous Media 1(1):3-25. doi:10.1007/ BF01036523

67. Whitaker S (1999) The method of, vol averaging. Kluwer Academic Publishers, Dordrecht

68. Yang J, Jia Y, Sun S, Ma D, Shi T, An L (2006) Mesoscopic simulation of the impregnating process of unidirectional fibrous preform in resin transfer molding. Mater Sci Eng A 435-436:515520. doi:10.1016/j.msea.2006.07.110

69. Zhang X, Zhang X (2003) Exact integration in the boundary element method for two-dimensional elastostatic problems. Eng Anal Bound Elem 27(10):987-997. doi:10.1016/ S0955-7997(03)00091-2

70. Zhdanov VG, Starov VM (2002) Calculation of the effective properties of porous and composite materials. Colloid J 64(6):706-715 